\title{
Dark matter and collider signals in an MSSM extension with vector-like multiplets
}

\author{
Jack Y. Araz, ${ }^{1, *}$ Shankha Banerjee, ${ }^{2, \dagger}$ Mariana Frank, ${ }^{1, \ddagger}$ Benjamin Fuks, ${ }^{3,4,}$ and Andreas Goudelis ${ }^{3,5, \|}$ \\ ${ }^{1}$ Concordia University 7141 Sherbrooke Street West, Montreal, Quebec, Canada H4B 1R6 \\ ${ }^{2}$ Institute for Particle Physics Phenomenology, Department of Physics, \\ Durham University, Durham DH1 3LE, United Kingdom \\ ${ }^{3}$ Sorbonne Université, CNRS, Laboratoire de Physique Théorique et Hautes Énergies, LPTHE, F-75252 Paris, France \\ ${ }^{4}$ Institut Universitaire de France, 103 boulevard Saint-Michel, 75005 Paris, France \\ ${ }^{5}$ Sorbonne Université, Institut Lagrange de Paris (ILP), 75014 Paris, France
}

(Received 23 October 2018; published 5 December 2018)

\begin{abstract}
Motivated by grand unification considerations, we analyse a simple extension of the minimal supersymmetric standard model with additional pairs of vector-like chiral supermultiplets. We focus on the so-called LND setup, which enlarges the particle content of the minimal model by two vector-like pairs of weak doublets (one pair of leptons and one pair of down-type quarks) and one vector-like pair of neutrino singlets. Imposing collider and low-energy constraints, sneutrinos and neutralinos both emerge as possible lightest supersymmetric particles and thus dark matter candidates. We perform a complete analysis of the dark sector and study the viability of these neutralino and sneutrino dark matter options. We show that cosmological considerations (the dark matter relic abundance and its direct and indirect detection signals) restrict neutralino dark matter to exhibit similar properties as in the minimal supersymmetric standard model, and impose the sneutrino dark matter candidate to be singlet-like, rather than doublet-like. Allowing the mixing of the fermionic component of the new supermultiplets with the Standard Model third generation fermions, we moreover demonstrate the existence of collider signals that are distinguishable from other, more minimal, supersymmetric scenarios by virtue of an enhanced production of events enriched in tau leptons. We furthermore show that this signature yields robust LHC signals, that could potentially be differentiated from the background in future data.
\end{abstract}

DOI: 10.1103/PhysRevD.98.115009

\section{INTRODUCTION}

During the past few decades, supersymmetry has gained the status of one of the best theoretically motivated scenarios for physics beyond the standard model (SM). Under specific conditions it can address the hierarchy problem, achieve gauge coupling unification and, when $R$-parity is conserved, provide natural dark matter candidate(s). However, supersymmetric models of particle physics have been under assault both from collider searches and from direct and indirect dark matter detection experiments. The measured value of the Higgs-boson mass seems to

\footnotetext{
*jack.araz@concordia.ca

shankha.banerjee@durham.ac.uk

*mariana.frank@concordia.ca

${ }^{\S}$ fuks@lpthe.jussieu.fr

"andreas.goudelis@1pthe.jussieu.fr
}

Published by the American Physical Society under the terms of the Creative Commons Attribution 4.0 International license. Further distribution of this work must maintain attribution to the author(s) and the published article's title, journal citation, and DOI. Funded by SCOAP. require $\mathrm{TeV}$-scale scalar quarks, a fact further strengthened by the results of direct sparticle searches at the LHC. The null results from dark matter searches have put substantial pressure on (light) neutralino dark matter and, since long ago, have wiped out left-handed sneutrinos as phenomenologically viable dark matter candidates. Furthermore, in its minimal version, supersymmetry fails to explain neutrino masses. Before, however, abandoning low-scale supersymmetry, one may ask if some of these outstanding issues can be addressed in extensions of the minimal supersymmetric standard model (MSSM), while maintaining the attractive features of the latter and giving rise to novel signals at colliders and elsewhere.

A large variety of MSSM extensions have been studied in the past, including (but not limited to) effective approaches $[1,2]$, as well as minimal modifications of the MSSM particle content $[3,4]$ or gauge group structure [5,6]. In Ref. [7], a less minimal approach was proposed, extending the MSSM particle content by additional pairs of vector-like supermultiplets. The advantage of this choice is that the Higgsboson mass can be raised while maintaining perturbative gauge coupling unification. The suggested models involve either $\mathbf{5}+\overline{\mathbf{5}}$ complete representations of $S U(5)$ (the 'LND' 
scenario) or $\mathbf{1 0}+\overline{\mathbf{1 0}}$ complete representations of $S U(5)$ (the 'QUE' scenario). Additionally, a 'QDEE' setup which does not contain complete multiplets of $S U(5)$ but still leads to gauge coupling unification has also been envisaged. In this notation scheme, the capital letters denote the nature of the extra supermultiplets relatively to their MSSM counterparts carrying the same charge, color and $B-L$ quantum numbers.

The dark matter phenomenology of the QUE and QDEE models was already studied in Refs. [8,9]. In both cases, the dark matter candidate is the lightest neutralino, much like in the MSSM, albeit with some interesting twists, and their phenomenology is rather similar. In this work we will focus on the third scenario, the so-called LND model. Although in this scenario the little hierarchy problem of the MSSM cannot be resolved [7], the LND model presents some other attractive features. As above-mentioned, it can lead to gauge coupling unification, although this necessitates that the vector-like fermions have masses in the $600-1000 \mathrm{GeV}$ window [7]. The field content contains a pair of vector-like neutrino singlets, whose fermionic components can be seen as a sterile neutrino. This could consequently provide explanations for the hints of neutrino oscillations at a higher frequency and for the differences between the neutrinos and antineutrinos measured by the LSND and MiniBooNE experiments [10,11]. The model moreover features two potential dark matter candidates, the lightest neutralino as well as the lightest singlet-like sneutrino. Furthermore, under a specific configuration, it could give rise to additional contributions to the anomalous magnetic moment of the muon, and large mixings between the new fermions and the third generation SM fermions are allowed and can lead to distinctive signals at the LHC. With this as motivation, we expect the phenomenology of this model to differ from that of the QUE and QDEE models, and in this paper we perform an analysis of the dark matter constraints and collider implications for this model.

This paper is structured as follows. In Sec. II we present the superfield content of the LND model, its superpotential and soft supersymmetry-breaking Lagrangian, and detail the particle mixings that are relevant for dark matter. In Sec. III we describe the setup of our parameter space exploration, and provide information on the experimental constraints that are imposed within our scan and the computational tools that have been employed. In Sec. IV we study the dark matter phenomenology of our model, separately for the case of a neutralino and a sneutrino LSP. The consequences of the model at the LHC are explored in Sec. V. Finally, we conclude in Sec. VI.

\section{THEORETICAL FRAMEWORK}

\section{A. Field content and Lagrangian}

The LND model is an extension of the MSSM inspired by $S U(5)$ grand unification (GUT) considerations.
We begin with the MSSM chiral superfield content that contains three generations of quark $(Q)$ and lepton $(L)$ weak doublets, as well as three generations of up-type quark $(\bar{U})$, down-type quark $(\bar{D})$ and charged lepton $(\bar{E})$ weak singlets. In our notations, the fermionic and scalar components of these supermultiplets read

$$
\begin{aligned}
& Q \equiv\left(q_{L}, \tilde{q}_{L}\right) \sim\left(\mathbf{3}, \mathbf{2}, \frac{1}{6}\right), \\
& L \equiv\left(\ell_{L}, \tilde{\ell}_{L}\right) \sim\left(\mathbf{1}, \mathbf{2},-\frac{1}{2}\right), \\
& \bar{U} \equiv\left(u_{R}^{c}, \tilde{u}_{R}^{\dagger}\right) \sim\left(\overline{\mathbf{3}}, \mathbf{1},-\frac{2}{3}\right), \\
& \bar{D} \equiv\left(d_{R}^{c}, \tilde{d}_{R}^{\dagger}\right) \sim\left(\overline{\mathbf{3}}, \mathbf{1}, \frac{1}{3}\right), \\
& \bar{E} \equiv\left(e_{R}^{c}, \tilde{e}_{R}^{\dagger}\right) \sim(\mathbf{1}, \mathbf{1}, 1),
\end{aligned}
$$

where we also indicate their representation under the $G_{\mathrm{MSSM}} \equiv S U(3)_{c} \times S U(2)_{L} \times U(1)_{Y}$ gauge group. The ${ }^{c}$ superscript indicates charge conjugation while the ${ }_{L, R}$ subscripts refer to the left- and right-handedness of the fermion.

In the model considered in this work, the MSSM matter sector of Eq. (2.1) is extended by vector-like pairs of supermultiplets forming a complete $\mathbf{5} \oplus \overline{\mathbf{5}}$ representation of $S U(5)$. Such a configuration allows to keep a reasonable level of simplicity and to maintain perturbative gauge coupling unification at high energy, with new states appearing at the $\mathrm{TeV}$ scale [12-15]. Decomposing the $\mathbf{5} \oplus$ $\overline{5}$ GUT supermultiplets in terms of the $G_{\mathrm{MSSM}}$ gauge group, the chiral content of the model includes one pair of vectorlike leptons $\left(L_{5}, \bar{L}_{5}\right)$ in the fundamental representation of $S U(2)_{L}$ and one pair of vector-like down-type quarks $\left(D_{5}, \bar{D}_{5}\right)$ in the trivial representation of $S U(2)_{L}$,

$$
\begin{aligned}
& L_{5} \equiv\left(\ell_{5 L}, \tilde{\ell}_{5 L}\right) \sim\left(\mathbf{1}, \mathbf{2},-\frac{1}{2}\right), \\
& \bar{L}_{5} \equiv\left(\ell_{5 R}^{c}, \tilde{\ell}_{5 R}^{\dagger}\right) \sim\left(\mathbf{1}, \mathbf{2}, \frac{1}{2}\right), \\
& D_{5} \equiv\left(d_{5 L}, \tilde{d}_{5 L}\right) \sim\left(\mathbf{3}, \mathbf{1},-\frac{1}{3}\right), \\
& \bar{D}_{5} \equiv\left(d_{5 R}^{c}, \tilde{d}_{5 R}^{\dagger}\right) \sim\left(\overline{\mathbf{3}}, \mathbf{1}, \frac{1}{3}\right) .
\end{aligned}
$$

As in many GUT-inspired supersymmetric models, we also add a pair of vector-like gauge singlets, ${ }^{1}$

$N \equiv\left(N_{L}, \tilde{N}_{L}\right) \sim(\mathbf{1}, \mathbf{1}, 0), \quad \bar{N} \equiv\left(N_{R}^{c}, \tilde{N}_{R}^{\dagger}\right) \sim(\mathbf{1}, \mathbf{1}, 0)$,

that can be mapped to a pair of extra (s)neutrinos and find motivation in dark matter and neutrino physics [16].

\footnotetext{
${ }^{1}$ This choice is consistent with minimal representations which do not violate lepton number.
} 
With respect to the MSSM, the model features one extra down-type quark and two extra down-type squarks, one extra charged lepton and two additional charged sleptons as well as two more neutrinos along with their accompanying four extra sneutrinos. The Higgs sector is identical to the MSSM one and consists of two weak doublets of Higgs supermultiplets

$$
\begin{aligned}
& H_{d} \equiv\left(\tilde{H}_{d}, h_{d}\right) \sim\left(\mathbf{1}, 2,-\frac{1}{2}\right), \\
& H_{u} \equiv\left(\tilde{H}_{u}, h_{u}\right) \sim\left(\mathbf{1}, 2, \frac{1}{2}\right),
\end{aligned}
$$

that are sufficient to break $G_{\mathrm{MSSM}}$ down to $U(1)_{\mathrm{em}}$ and generate supersymmetric masses for all particles. Finally, the model includes three gauge supermultiplets (as the gauge group is the same as in the MSSM) that we denote by

$$
\begin{aligned}
G & \equiv\left(g, \lambda_{\tilde{G}}\right) \sim(\mathbf{8}, \mathbf{1}, 0), \\
W & \equiv\left(w, \lambda_{\tilde{W}}\right) \sim(\mathbf{1}, \mathbf{3}, 0), \\
B & \equiv\left(b, \lambda_{\tilde{B}}\right) \sim(\mathbf{1}, \mathbf{1}, 0),
\end{aligned}
$$

for the QCD $(G)$, weak $(W)$ and hypercharge $(B)$ gauge groups.

The supersymmetry-conserving (nongauge) interactions of the model are driven by the superpotential $W_{\mathrm{LND}}$ that is written, assuming $R$-parity conservation, as [7]

$$
\begin{aligned}
W_{\mathrm{LND}}= & \mu H_{u} \cdot H_{d}+\mathbf{y}_{\mathbf{u}} \bar{U} Q \cdot H_{u}-\mathbf{y}_{\mathbf{d}} \bar{D} Q \cdot H_{d}-\mathbf{y}_{\mathbf{e}} \bar{E} L \cdot H_{d} \\
& +\mu_{D} D_{5} \bar{D}_{5}+\mu_{L} L_{5} \cdot \bar{L}_{5}+\mu_{N} N \bar{N}+k_{N} \bar{N} L_{5} \cdot H_{u} \\
& -h_{N} N \bar{L}_{5} \cdot H_{d}-\varepsilon_{\mathbf{D}} \bar{D}_{5} Q \cdot H_{d}-\varepsilon_{\mathbf{E}} \bar{E} L_{5} \cdot H_{d} \\
& +\varepsilon_{\mathbf{N}} \bar{N} L \cdot H_{u}+\kappa_{\mathbf{D}} D_{5} \bar{D}+\kappa_{\mathbf{L}} L \cdot \bar{L}_{5},
\end{aligned}
$$

where all flavor indices have been explicitly omitted for simplicity. The first terms correspond to the MSSM superpotential in which $\mu$ denotes the MSSM off-diagonal Higgs(ino) mass contribution and $\mathbf{y}_{\mathbf{u}}, \mathbf{y}_{\mathbf{d}}$ and $\mathbf{y}_{\mathbf{e}}$ stand for the up-type quark, down-type quark and charged lepton Yukawa matrices in flavor space. Moreover, $\mu_{D}, \mu_{L}$ and $\mu_{N}$ are explicit masses for the non-MSSM fields and $h_{N}$ and $k_{N}$ stand for the Yukawa interactions of the noncolored vector-like superfields. The terms of the last line of the superpotential include new Yukawa couplings driving the mixing of the standard model fermions with their vector-like counterparts (the $\varepsilon_{\mathbf{D}}, \varepsilon_{\mathbf{E}}$ and $\varepsilon_{\mathbf{N}}$ vectors in flavor space) as well as direct mass mixing terms (the $\kappa_{\mathbf{D}}$ and $\kappa_{\mathbf{L}}$ vectors in flavor space). While these are strongly constrained by flavor data, such mixings have to be nonvanishing to prevent the existence of unwanted cosmologically stable relics. For consistency with both flavor and cosmology constraints, in the following we will assume the existence of a small mixing with the third generation of standard model fermions only.

As in any realistic supersymmetric model, supersymmetry has to be softly broken. The Lagrangian thus includes gaugino and scalar mass terms, as well as bilinear and trilinear scalar interactions whose form is obtained from the superpotential. The gaugino mass contributions read

$$
\mathcal{L}_{\text {soft }}^{(\lambda)}=-\frac{1}{2}\left(M_{1} \lambda_{\tilde{B}} \cdot \lambda_{\tilde{B}}+M_{2} \lambda_{\tilde{W}} \cdot \lambda_{\tilde{W}}+M_{3} \lambda_{\tilde{g}} \cdot \lambda_{\tilde{g}}+\text { H.c. }\right),
$$

where the $M_{1}, M_{2}$ and $M_{3}$ parameters represent the bino, wino and gluino masses, and the scalar mass Lagrangian is given by

$$
\begin{aligned}
\mathcal{L}_{\text {soft }}^{(\phi)}= & -m_{H_{d}}^{2} h_{d}^{\dagger} h_{d}-m_{H_{u}}^{2} h_{u}^{\dagger} h_{u}-\mathbf{m}_{\tilde{\mathbf{Q}}}^{2} \tilde{q}_{L}^{\dagger} \tilde{q}_{L}-\mathbf{m}_{\tilde{\mathbf{d}}}^{2} \tilde{d}_{R}^{\dagger} \tilde{d}_{R}-\mathbf{m}_{\tilde{\mathbf{u}}}^{2} \tilde{u}_{R}^{\dagger} \tilde{u}_{R}-\mathbf{m}_{\tilde{\mathbf{L}}}^{2} \tilde{\ell}_{L}^{\dagger} \tilde{\ell}_{L}-\mathbf{m}_{\tilde{\tilde{\mathbf{e}}}}^{2} \tilde{e}_{R}^{\dagger} \tilde{e}_{R}-m_{\tilde{L}_{5}}^{2} \tilde{\ell}_{5 L}^{\dagger} \tilde{\ell}_{5 L}-m_{\tilde{L}_{5}}^{2} \tilde{\ell}_{5 R}^{\dagger} \tilde{\ell}_{5 R} \\
& -m_{\tilde{D}_{5}}^{2} \tilde{d}_{5 R}^{\dagger} \tilde{d}_{5 R}-m_{\tilde{\tilde{D}}_{5}}^{2} \tilde{d}_{5 L}^{\dagger} \tilde{d}_{5 L}-m_{\tilde{N}}^{2} \tilde{N}_{L}^{\dagger} \tilde{N}_{L}-m_{\tilde{\tilde{N}}_{R}}^{2} \tilde{N}_{R}^{\dagger} \tilde{N}_{R}-\left[\mathbf{m}_{\tilde{\mathbf{L}}_{5}, \tilde{\mathbf{L}}}^{2} \tilde{\ell}_{L}^{\dagger} \tilde{\ell}_{5 L}+\text { H.c. }\right]-\left[\mathbf{m}_{\tilde{\mathbf{D}}_{5}, \tilde{\mathbf{D}}}^{2} \tilde{d}_{R}^{\dagger} \tilde{d}_{5 R}+\text { H.c. }\right],
\end{aligned}
$$

where $m_{i}^{2}$ represent the various mass parameters, in flavor space. Moreover, the superpotential-induced soft terms are written as

$$
\begin{aligned}
\mathcal{L}_{\text {soft }}^{(W)}= & {\left[-b h_{u} \cdot h_{d}+\mathbf{T}_{d} \tilde{d}^{\dagger} \tilde{q} \cdot h_{d}+\mathbf{T}_{e} \tilde{e}^{\dagger} \tilde{l} \cdot h_{d}-\mathbf{T}_{u} \tilde{u}^{\dagger} \tilde{d} \cdot h_{u}\right.} \\
& -b_{D} \tilde{d}_{5 R}^{\dagger} \tilde{d}_{5 L}-b_{L} \tilde{\ell}_{5 R}^{\dagger} \cdot \tilde{\ell}_{5 L}-b_{N} \tilde{N}_{R}^{\dagger} \tilde{N}_{L}-a_{k_{N}} \tilde{N}_{R}^{\dagger} \tilde{\ell}_{5 L} \\
& \cdot h_{u}+a_{h_{N}} \tilde{N}_{L} \tilde{\ell}_{5 R}^{\dagger} \cdot h_{d}+\mathbf{a}_{\varepsilon_{\mathbf{D}}} \tilde{d}_{5 R}^{\dagger} \tilde{q}_{L} \cdot h_{d}+\mathbf{a}_{\varepsilon_{\mathbf{E}}} \tilde{e}_{R}^{\dagger} \tilde{\ell}_{5 L} \\
& \left.\cdot h_{d}-\mathbf{a}_{\varepsilon_{\mathrm{N}}} \tilde{N}_{R}^{\dagger} \tilde{\ell}_{L} \cdot h_{u}-\mathbf{b}_{\kappa_{\mathbf{D}}} \tilde{d}_{R}^{\dagger} \tilde{d}_{5 L}-\mathbf{b}_{\kappa_{\mathbf{L}}} \tilde{\ell}_{5 R}^{\dagger} \cdot \tilde{\ell}_{L}+\text { H.c. }\right],
\end{aligned}
$$

where the first four terms are the usual MSSM soft terms, $b$ denotes the bilinear Higgs interaction strength and $\mathbf{T}_{\mathbf{i}}$ the various squark-Higgs trilinear interactions in flavor space. The $a_{k_{N}}$ and $a_{h_{N}}$ parameters represent the trilinear couplings of the vector-like sneutrinos and sleptons to the Higgs fields, whilst the $\mathbf{a}_{\varepsilon_{\mathrm{i}}}$ and $\mathbf{b}_{\kappa_{\mathrm{i}}}$ parameters are three-dimensional vectors describing the mixing of the vector-like and MSSM scalars. Similarly to their superpotential term counterparts, the latter will be assumed vanishing for the first two generations, and small for the third generation.

Once electroweak symmetry is broken, all particles with the same electric charge and lying in the same color and spin representations mix. The neutralino sector is identical to the MSSM one, consisting of four Majorana fermions $\chi_{i}^{0}, i=1, \ldots, 4$, which are linear combinations of the four neutral gaugino and Higgsino gauge eigenstates. The lightest neutralino, $\chi_{1}^{0}$ is the first dark matter candidate we shall consider. The model also contains five physical neutrinos which are admixtures of the usual MSSM neutrinos with the fermionic neutral components 
of $L_{5}$ and $\bar{L}_{5}$ as well as with the fermionic components of the gauge singlet chiral superfields $N$ and $\bar{N}$. The two heaviest exotic states are not stable, since they can decay through the Yukawa-like $\varepsilon_{\mathbf{E}}$ and $\varepsilon_{\mathbf{N}}$ terms in Eq. (2.6), and thus they cannot be potential dark matter candidates. The second dark matter candidate considered is in the sneutrino sector, which in the LND model consists of seven physical scalars. The sneutrino mixing is described by the symmetric mass matrix $M_{\tilde{\nu}}^{2}$. In the $\left(\tilde{\nu}_{5 L}, \tilde{N}_{L}\right.$, $\left.\tilde{\nu}_{e}, \tilde{\nu}_{\mu}, \tilde{\nu}_{\tau}, \tilde{\nu}_{5 R}^{\dagger}, \tilde{N}_{R}^{\dagger}\right)$ basis, matrix elements in the $7 \times 7$ symmetric matrix include soft terms, supersymmetric contributions as well as $D$-term contributions and are given by

$$
\begin{aligned}
& \left(M_{\tilde{\nu}}^{2}\right)_{11}=\frac{1}{8}\left[\frac{e^{2}}{s_{w}^{2}}+\frac{e^{2}}{c_{w}^{2}}\right]\left[v_{d}^{2}-v_{u}^{2}\right]+\mu_{L}^{2}+m_{\tilde{L}_{5}}^{2}+\frac{1}{2} k_{N}^{2} v_{u}^{2}, \\
& \left(M_{\tilde{\nu}}^{2}\right)_{12}=\frac{1}{\sqrt{2}}\left[h_{N} \mu_{L} v_{d}+k_{N} \mu_{N} v_{u}\right], \\
& \left(M_{\tilde{\nu}}^{2}\right)_{1(2+f)}=\mu_{L}\left(\kappa_{\mathbf{L}}\right)_{f}+\frac{1}{2} k_{N} v_{u}^{2}\left(\varepsilon_{\mathbf{N}}\right)_{f}+\left(\mathbf{m}_{\tilde{\mathbf{L}}_{5}, \mathbf{L}}^{2}\right)_{f}, \\
& \left(M_{\tilde{\nu}}^{2}\right)_{16}=b_{L}, \\
& \left(M_{\tilde{\nu}}^{2}\right)_{17}=\frac{1}{\sqrt{2}}\left[a_{k_{N}} v_{u}-\mu k_{N} v_{d}\right], \\
& \left(M_{\tilde{\nu}}^{2}\right)_{22}=\mu_{N}^{2}+m_{\tilde{N}}^{2}+\frac{1}{2} h_{N}^{2} v_{d}^{2}, \\
& \left.\left(M_{\tilde{\nu}}^{2}\right)_{2(2+f)}=\mu_{N} v_{u}\left(\varepsilon_{\mathbf{N}}\right)_{f}+h_{N} v_{d}\left(\kappa_{\mathbf{L}}\right)_{f}\right] \text {, } \\
& \left(M_{\tilde{\nu}}^{2}\right)_{26}=\frac{1}{\sqrt{2}}\left[a_{h_{N}} v_{d}-\mu h_{N} v_{u}\right], \\
& \left(M_{\tilde{\nu}}^{2}\right)_{27}=b_{N} \text {, } \\
& \left(M_{\tilde{\nu}}^{2}\right)_{(2+f)\left(2+f^{\prime}\right)}=\frac{1}{8}\left[\frac{e^{2}}{s_{w}^{2}}+\frac{e^{2}}{c_{w}^{2}}\right]\left[v_{d}^{2}-v_{u}^{2}\right] \delta_{f f^{\prime}}+\left(\mathbf{m}_{\tilde{\mathbf{L}}}^{2}\right)_{f f^{\prime}} \\
& +\frac{1}{2} v_{u}^{2}\left(\varepsilon_{\mathbf{N}}\right)_{f}\left(\varepsilon_{\mathbf{N}}\right)_{f^{\prime}}+\left(\kappa_{\mathbf{L}}\right)_{f}\left(\kappa_{\mathbf{L}}\right)_{f^{\prime}}, \\
& \left(M_{\tilde{\nu}}^{2}\right)_{(2+f) 7}=\frac{1}{\sqrt{2}}\left[v_{u}\left(\mathbf{a}_{\varepsilon_{\mathbf{N}}}\right)_{f}-\mu v_{d}\left(\varepsilon_{\mathbf{N}}\right)_{f}\right] \text {, } \\
& \left(M_{\tilde{\nu}}^{2}\right)_{66}=\frac{1}{8}\left[\frac{e^{2}}{s_{w}^{2}}+\frac{e^{2}}{c_{w}^{2}}\right]\left[v_{u}^{2}-v_{d}^{2}\right]+\mu_{L}^{2}+m_{\tilde{L}_{5}}^{2}+\frac{1}{2} h_{N}^{2} v_{d}^{2} \\
& +\sum_{f=1}^{3}\left(\kappa_{\mathbf{L}}\right)_{f}^{2} \\
& \left(M_{\tilde{\nu}}^{2}\right)_{67}=\frac{1}{\sqrt{2}}\left[h_{N} v_{d} \mu_{N}+k_{N} v_{u} \mu_{L}\right. \\
& \left.+v_{u} \sum_{f=1}^{3}\left(\kappa_{\mathbf{L}}\right)_{f}\left(\varepsilon_{\mathbf{N}}\right)_{f}\right] \text {, } \\
& \left(M_{\tilde{\nu}}^{2}\right)_{77}=\mu_{N}^{2}+m_{\tilde{\tilde{N}}}^{2}+\frac{1}{2} k_{N}^{2} v_{u}^{2}+\frac{1}{2} v_{u} \sum_{f=1}^{3}\left(\varepsilon_{\mathbf{N}}\right)_{f}^{2},
\end{aligned}
$$

where $f, f^{\prime}=1,2$ and 3 are generation indices, $\left(M_{\tilde{\nu}}^{2}\right)_{i j}=$ $\left(M_{\tilde{\nu}}^{2}\right)_{j i}$ and all other elements vanish.

\section{PARAMETER SPACE EXPLORATION}

\section{A. Parameter space}

As indicated in the Lagrangian introduced in the previous section, the LND model parameter space is defined from a large set of beyond the SM free parameters. Assuming unification conditions and relying on the minimization of the scalar potential, this list can be further reduced. In the following, we define the range of value allowed for each parameter relevant for our study. We have verified that wider ranges did not yield any new phenomenology. We fixed the values of all input parameters at the supersymmetry-breaking scale, with the exception of the common MSSM sfermion and Higgs mass $M_{0}$ and their common soft trilinear coupling $A_{0}$ that are defined at the GUT-scale. This particular choice allows us to analyze a large set of different scalar spectra, concentrating in particular on scenarios with light electroweak scalars and heavier strongly-interacting ones (that are only marginally relevant for our dark matter study). For the supersymmetry-breaking scale we have taken the geometric mean of the masses of the lightest $\left(M_{\tilde{u}_{1}}\right)$ and heaviest $\left(M_{\tilde{u}_{6}}\right)$ up-type squarks, $M_{\text {SUSY }} \sim \sqrt{M_{\tilde{u}_{1}} M_{\tilde{u}_{6}}}$, and restricted it to be smaller than $5 \mathrm{TeV}$.

We start with the superpotential parameters appearing in Eq. (2.6). While the SM first and second generation Yukawa couplings are neglected, we fix the third generation ones to the value given in the Particle Data Group Review [17]. All other parameters are left free and will be scanned over, with the exception of the off-diagonal Higgs mixing parameter $\mu$ whose absolute value is fixed from the scalar potential minimization conditions. The supersymmetric masses of the three pairs of vector-like supermultiplets $\mu_{D}, \mu_{L}$ and $\mu_{N}$ are taken as varying in the $\mathrm{GeV}-\mathrm{TeV}$ range,

$\mu_{D} \in[1,8] \mathrm{TeV}, \quad \mu_{L} \in[0,3] \mathrm{TeV}$ and

$\mu_{N} \in[0,5] \mathrm{TeV}$,

whilst the vector-like Yukawa couplings are taken of $\mathcal{O}(1)$,

$$
k_{N} \in[-1,1] \text { and } h_{N} \in[-1,1] .
$$

As previously stated, we forbid any mixing between the vector-like sector and the first two SM generations, so that the supersymmetric mass mixing parameters and $\varepsilon$ Yukawa couplings solely involve the third generation,

$$
\begin{aligned}
& \kappa_{\mathbf{D}}=\left(\begin{array}{c}
0 \\
0 \\
\kappa_{D}
\end{array}\right), \quad \kappa_{\mathbf{L}}=\left(\begin{array}{c}
0 \\
0 \\
\kappa_{L}
\end{array}\right), \quad \varepsilon_{\mathbf{D}}=\left(\begin{array}{c}
0 \\
0 \\
\varepsilon_{D}
\end{array}\right), \\
& \varepsilon_{\mathbf{E}}=\left(\begin{array}{c}
0 \\
0 \\
\varepsilon_{E}
\end{array}\right) \quad \text { and } \quad \varepsilon_{\mathbf{N}}=\left(\begin{array}{c}
0 \\
0 \\
\varepsilon_{N}
\end{array}\right),
\end{aligned}
$$


with

$\kappa_{D} \in\left[-10^{-6}, 10^{-6}\right] \mathrm{TeV}, \quad \kappa_{L} \in[-1,1] \mathrm{TeV}$,

$\varepsilon_{N} \in[-0.1,0.1], \quad \varepsilon_{E} \in[-1,1] \quad$ and

$\varepsilon_{D} \in[-5,5] \times 10^{-3}$.

The parameters in the down-type quark sector are restricted to be small by flavor constraints. The quoted intervals have been determined after scanning over larger ranges and restricting the parameters responsible for flavor-changing effects in the down-type quark and charged lepton sectors according to the constraints in Table II. The soft gaugino mass terms of Eq. (2.7) are allowed to vary independently in the $\mathrm{GeV}$-multi-TeV range,

$M_{1} \in[0,2] \mathrm{TeV}, \quad M_{2} \in[0,3] \mathrm{TeV} \quad$ and

$M_{3} \in[0,4] \mathrm{TeV}$,

whilst the MSSM squark and Higgs mass parameters appearing in Eq. (2.8) are imposed to unify to a common $M_{0}$ value,

$$
M_{0} \in[0,5] \mathrm{TeV} .
$$

The extra squark mass and mixing parameters are chosen to vary independently,

$$
\begin{aligned}
& m_{\tilde{D}_{5}} \in[0,5] \mathrm{TeV}, \quad m_{\tilde{D}_{5}} \in[0,5] \mathrm{TeV}, \\
& \mathbf{m}_{\tilde{\mathbf{D}}_{5}, \tilde{\mathbf{D}}}^{2}=\left(\begin{array}{c}
0 \\
0 \\
m_{\tilde{D}_{5}, \tilde{D}}^{2}
\end{array}\right) \text { with } \\
& m_{\tilde{D}_{5}, \tilde{D}}^{2} \in[-0.1,0.1] \mathrm{TeV}^{2} .
\end{aligned}
$$

These new states introduce some of the specific features of the LND model, but our dark matter analysis and collider signals are largely independent of this choice. By contrast, the mass parameters of the extra sneutrinos and sleptons are also specific parameters of the model, but very relevant for what concerns cosmology. We keep all of them free and allow them to vary independently, again in the multi- $\mathrm{TeV}$ range,

$$
\begin{aligned}
& m_{\tilde{N}} \in[0,6] \mathrm{TeV}, \quad m_{\tilde{\tilde{N}}} \in[0,6] \mathrm{TeV}, \\
& m_{\tilde{L}_{5}} \in[0,1] \mathrm{TeV} \quad \text { and } \quad m_{\tilde{L}_{5}} \in[0,1] \mathrm{TeV},
\end{aligned}
$$

and we fix the mass mixing between the MSSM and the vector-like sleptons as

$$
\mathbf{m}_{\tilde{\mathbf{L}}_{5}, \tilde{\mathbf{L}}}^{2}=\left(\begin{array}{c}
0 \\
0 \\
m_{\tilde{L}_{5}, \tilde{L}}^{2}
\end{array}\right) \quad \text { with } \quad m_{\tilde{L}_{5}, \tilde{L}}^{2} \in[-0.1,0.1] \mathrm{TeV}^{2} .
$$

The rest of the soft parameters involving the MSSM sfermions and Higgs bosons are assumed to unify at the GUT scale, so that all squark trilinear couplings to the Higgs sector are set to a common $A_{0}$ value multiplied by the relevant SM fermion Yukawa coupling. In addition, all trilinear scalar couplings involving two vector-like sfermions are taken vanishing. Moreover, all bilinear terms are fixed to a common $B_{0}$ value, with the exception of the Higgs mixing parameter $b$ whose value is driven by the scalar potential minimization. We thus choose

$$
\begin{aligned}
B_{0} & \in[-5,5] \mathrm{TeV}, \quad A_{0} \in[-2,2] \quad \text { and } \\
a_{k_{N}} & =a_{h_{N}}=0 .
\end{aligned}
$$

As for all other interactions involving the mixing of a vectorlike and an MSSM particle, we enforce the soft ones to vanish for the first two generations,

$\mathbf{a}_{\varepsilon_{\mathbf{D}}}=\left(\begin{array}{c}0 \\ 0 \\ a_{\varepsilon_{D}}\end{array}\right), \quad \mathbf{a}_{\varepsilon_{\mathbf{E}}}=\left(\begin{array}{c}0 \\ 0 \\ a_{\varepsilon_{E}}\end{array}\right) \quad$ and $\quad \mathbf{a}_{\varepsilon_{\mathrm{N}}}=\left(\begin{array}{c}0 \\ 0 \\ a_{\varepsilon_{N}}\end{array}\right)$,

and, for simplicity, fix the input values of the three remaining free parameters to zero,

$$
a_{\varepsilon_{D}}=a_{\varepsilon_{E}}=a_{\varepsilon_{N}}=0 .
$$

Since the SM Higgs-boson mass is taken equal to its measured value, the Higgs sector is fully defined from the conditions stemming from the minimization of the scalar potential, once one extra parameter is fixed, as all parameters contributing at the one-loop order and beyond are already defined above. The ratio of the vacuum expectation values of the neutral components of the two Higgs doublets, $\tan \beta$, is allowed to vary in the following range,

$$
\tan \beta \in[1,60] .
$$

The parameter space is now defined by the 25 new physics parameters and one sign is listed in Table I, where we summarize the free parameters and the range over they are scanned.

\section{B. Analysis setup and experimental constraints}

In order to explore the parameter space defined in Sec. III A, we have implemented the LND model in the SARAH 4.12.2 package [18], which we have used to generate the corresponding SPHENO (version 4.0.3) output [19]. With this last code, we derive the value of the model parameters at the electroweak scale through their renormalization group running from the input scale, and extract 
TABLE I. Range of the free parameters of the model scans. The SM parameters are fixed to the values reported in the Particle Data Group Review [17] and all nonlisted parameters are fixed to zero.

\begin{tabular}{lccc}
\hline \hline Parameter & Scanned range & Parameter & Scanned range \\
\hline$\mu_{D}$ & {$[1,8] \mathrm{TeV}$} & $M_{1}$ & {$[0,2] \mathrm{TeV}$} \\
$\mu_{N}$ & {$[0,5] \mathrm{TeV}$} & $M_{2}$ & {$[0,3] \mathrm{TeV}$} \\
$\mu_{L}$ & {$[0,3] \mathrm{TeV}$} & $M_{3}$ & {$[0,4] \mathrm{TeV}$} \\
$\operatorname{sgn}(\mu)$ & \pm 1 & $m_{\tilde{L}_{5}}, m_{\tilde{L}_{5}}$ & {$[0,1] \mathrm{TeV}$} \\
$h_{N}, k_{N}$ & {$[-1,1]$} & $m_{\tilde{D}_{5}}, m_{\tilde{D}_{5}}$ & {$[0,5] \mathrm{TeV}$} \\
$\kappa_{L}$ & {$[-1,1] \mathrm{TeV}$} & $m_{\tilde{N}}, m_{\tilde{N}}$ & {$[0,6] \mathrm{TeV}$} \\
$\kappa_{D}$ & {$[-1,1] \times 10^{-6} \mathrm{TeV}$} & $m_{\tilde{L}_{5}, \tilde{L}}^{2}, m_{\tilde{D}_{5}, \tilde{D}}^{2}$ & {$[-0.1,0.1] \mathrm{TeV}{ }^{2}$} \\
$\varepsilon_{N}$ & {$[-0.1,0.1]$} & $A_{0}$ & {$[-2,2] \mathrm{TeV}$} \\
$\varepsilon_{E}$ & {$[-1,1]$} & $B_{0}$ & {$[-5,5] \mathrm{TeV}$} \\
$\varepsilon_{D}$ & {$[-5,5] \times 10^{-3}$} & $\tan \beta$ & {$[1,60]$} \\
$M_{0}$ & {$[0,5] \mathrm{TeV}$} & & \\
\hline \hline
\end{tabular}

the particle spectrum. In order to assess the phenomenological viability of the different scenarios probed during the scan, we enforce the compatibility with several flavor, collider and low-energy physics observables calculated by SPHENO and summarized in Table II. The scenarios considered in our study must satisfy rare $B$-decay constraints [20-22],

$$
\begin{aligned}
\operatorname{BR}\left(B^{0} \rightarrow X_{s} \gamma\right) & \in[2.99,3.87] \times 10^{-4}, \\
\operatorname{BR}\left(B_{s}^{0} \rightarrow \mu^{+} \mu^{-}\right) & \in[1.1,6.4] \times 10^{-9} \text { and } \\
\frac{\operatorname{BR}\left(B \rightarrow \tau \nu_{\tau}\right)}{\operatorname{BR}_{\mathrm{SM}}\left(B \rightarrow \tau \nu_{\tau}\right)} & \in[0.15,2.41],
\end{aligned}
$$

rare tau-decay constraints [23-25],

$$
\begin{aligned}
& \operatorname{BR}(\tau \rightarrow e \gamma) \in[0,3.3] \times 10^{-8}, \\
& \operatorname{BR}(\tau \rightarrow \mu \gamma) \in[0,4.4] \times 10^{-8}, \\
& \operatorname{BR}(\tau \rightarrow e \pi) \in[0,8.0] \times 10^{-8}, \\
& \operatorname{BR}(\tau \rightarrow \mu \pi) \in[0,1.1] \times 10^{-7}, \\
& \operatorname{BR}(\tau \rightarrow 3 \mu) \in[0,2.1] \times 10^{-8},
\end{aligned}
$$

$B$-meson oscillation constraints [26],

$$
\Delta M_{s} \in[10.2,26.4] \mathrm{ps}^{-1}, \quad \Delta M_{d} \in[0.29,0.76] \mathrm{ps}^{-1},
$$

and flavor-violating Z-boson [27] and Higgs-boson [28] decay bounds,

$$
\begin{aligned}
& \operatorname{BR}(Z \rightarrow e \mu) \in[0,7.5] \times 10^{-7} \text { and } \\
& \operatorname{BR}(h \rightarrow e \mu) \in[0,3.5] \times 10^{-4} .
\end{aligned}
$$

Moreover, we impose the compatibility with electroweak precision observables (EWPO) at the $2 \sigma$ level [29], using a correlation function based on the oblique parameters $[30,31]$. Thanks to the interface of SPHENO with HiggsBounds version 4.3.1 [32] and HiggsSignals version 1.4.0 [33], we verify the consistency of the Higgs sector with experimental measurements of LHC Run 1. In practice, we check that the Higgs-boson mass, gluon and vector-boson fusion production cross sections (computed with the SUSHi program version 1.5 [34]) and signal strengths agree with data up to deviations corresponding to a global $\chi^{2}(\hat{\mu})$ quantity of at most 111.6, which corresponds to a $2 \sigma$ level of agreement for the number of considered observables.

TABLE II. Set of low-energy and flavor physics constraints imposed within our LND model scanning procedure (upper) and mass bounds imposed on the Higgs boson and new physics states (lower).

\begin{tabular}{lccc}
\hline \hline Observable & Constraint & Observable & Constraint \\
\hline $\operatorname{BR}\left(B^{0} \rightarrow X_{s} \gamma\right)$ & {$[2.99,3.87] \times 10^{-4}[20]$} & $\mathrm{BR}\left(B \rightarrow \tau \nu_{\tau}\right) / \mathrm{BR}_{\mathrm{SM}}\left(B \rightarrow \tau \nu_{\tau}\right)$ & {$[0.15,2.41][21]$} \\
$\operatorname{BR}\left(B_{s}^{0} \rightarrow \mu^{+} \mu^{-}\right)$ & {$[1.1,6.4] \times 10^{-9}[22]$} & $\mathrm{BR}(\tau \rightarrow e \gamma)$ & {$[0,3.3] \times 10^{-8}[23]$} \\
$\operatorname{BR}(\tau \rightarrow \mu \gamma)$ & {$[0,4.4] \times 10^{-8}[23]$} & $\mathrm{BR}(\tau \rightarrow e \pi)$ & {$[0,8.0] \times 10^{-8}[24]$} \\
$\mathrm{BR}(\tau \rightarrow \mu \pi)$ & {$[0,1.1] \times 10^{-7}[24]$} & $\mathrm{BR}(\tau \rightarrow 3 \mu)$ & {$[0,2.1] \times 10^{-8}[25]$} \\
$\Delta M_{s}$ & {$[10.2,26.4] \mathrm{ps}^{-1}[26]$} & $\Delta M_{d}$ & {$[0.29,0.76] \mathrm{ps}^{-1}[26]$} \\
$\mathrm{BR}(Z \rightarrow e \mu)$ & $\left.[0,7.5] \times 10^{-7}\right][27]$ & $\mathrm{BR}(h \rightarrow e \mu)$ & {$[0,3.5] \times 10^{-4}[28]$} \\
EWPO tests & $\leq 2 \sigma[29-31]$ & $\chi^{2}(\hat{\mu})$ & $\leq 111.6$ \\
\hline \hline Mass & Constraint & Mass & Constraint \\
\hline$M_{\chi_{2}^{0}}$ & $>62.4 \mathrm{GeV}[17]$ & $M_{\chi_{1}^{ \pm}}$ & $>103.5 \mathrm{GeV}[17]$ \\
$M_{\chi_{3}^{0}}$ & $>99.9 \mathrm{GeV}[17]$ & $M_{\tilde{e}}$ & $>107 \mathrm{GeV}[17]$ \\
$M_{\chi_{4}^{0}}$ & $>116 \mathrm{GeV}[17]$ & $M_{\tilde{\mu}}$ & $>94 \mathrm{GeV}[17]$ \\
$M_{\tilde{g}}$ & $>1.75 \mathrm{TeV}[35]$ & $M_{\tilde{\tau}}$ & $>81 \mathrm{GeV}[17]$ \\
$M_{\tilde{\tau}}$ & $>750 \mathrm{GeV}[36]$ & $M_{\tau^{\prime}}$ & $>103 \mathrm{GeV}[37]$ \\
$M_{h}$ & $125.09 \pm 3 \mathrm{GeV}[38]$ & & \\
\hline \hline
\end{tabular}


Additionally, we constrain superpartners masses (of the MSSM sector) from direct search bounds [17,35,36]. We impose that the gluino mass $\left(M_{\tilde{g}}\right)$, the neutralino and chargino masses $\left(M_{\tilde{\chi}_{i}^{0}}\right.$ and $\left.M_{\tilde{\chi}_{i}^{ \pm}}\right)$, the slepton masses $\left(M_{\tilde{e}}, M_{\tilde{\mu}}\right.$ and $\left.M_{\tilde{\tau}}\right)$ and the stop mass $\left(M_{\tilde{t}}\right)$ satisfy

$$
\begin{array}{rlrl}
M_{\tilde{g}} & >1.75 \mathrm{TeV}, & M_{\tilde{\chi}_{2}^{0}}>62.4 \mathrm{GeV}, \\
M_{\tilde{\chi}_{3}^{0}} & >99.9 \mathrm{GeV}, & M_{\tilde{\chi}_{4}^{0}} & >116 \mathrm{GeV}, \\
M_{\tilde{\chi}_{1}^{ \pm}} & >103.5 \mathrm{GeV}, & M_{\tilde{e}} & >107 \mathrm{GeV}, \\
M_{\tilde{\mu}} & >94 \mathrm{GeV}, & M_{\tilde{\tau}}>81 \mathrm{GeV}, \\
M_{\tilde{t}} & >750 \mathrm{GeV}, &
\end{array}
$$

and enforce the vector-like lepton mass $M_{\tau^{\prime}}$ to obey the LEP bound [37],

$$
M_{\tau^{\prime}}>103 \mathrm{GeV} .
$$

We now proceed to our analysis. We perform a scan of the parameter space by relying on the Metropolis-Hastings sampling method [39] in which the model free parameters vary as in Table I and are restricted by the constraints of Table II, with the additional requirement that the LSP has to be neutral. For each point, we perform the dark matter analysis with MICROMEGAs version 4.3.1 [40], which allows us to calculate all DM observables used in the analysis of Sec. IV from the LND CALCHEP [41] model file generated by SARAH. In Sec. V, we perform a collider analysis of a few benchmark scenarios representative of the different spectra favored by cosmology, by relying on the MG5_AMC@NLO [42] platform and an LND UFO model file [43] generated by SARAH. The interfacing of the various programmes and our numerical analysis have been performed with a modified version of the PYSLHA package version 3.1.1 [44].

\section{DARK MATTER PHENOMENOLOGY}

Having presented our model, the leading experimental constraints that we subject it to, and the methodology that we use in order to explore and assess the viability of the parameter space, we now proceed to present the results of our dark matter analysis. We divide the discussion into two parts, depending on the nature of the dark matter candidate (neutralino or sneutrino).

\section{A. Neutralino dark matter}

The lightest neutralino has, since long, been the most celebrated dark matter candidate of the MSSM. However, in the MSSM, barring coannihilations and funnels, the possibilities for neutralino dark matter with an $\mathcal{O}\left(10^{2}\right) \mathrm{GeV}$ mass are now severely constrained. In particular, almost pure Higgsinos and winos tend to be under-abundant, unless their mass lies above about $1 \mathrm{TeV}$, as the cosmologically-attractive possibility of a pure Higgsino with a mass below the $W$-boson mass $M_{W}$ is excluded from chargino searches at LEP [45-48]. Binos, on the other hand, tend to be overabundant by a few factors, unless either they can annihilate through the $t$-channel exchange of a sufficiently light sfermion into SM fermions, or they contain a substantial Higgsino or wino fraction. The former case is disfavored by sfermion searches at the LHC, whereas direct detection experiments [49] strongly constrain the mixed binoHiggsino scenario. The mixed bino-wino case is less constrained and constitutes one of the remaining possibilities for sub-TeV natural neutralino dark matter. ${ }^{2}$ We refer to Ref. [53] for a recent detailed account of existing constraints.

In the context of MSSM extensions with vector-like fermions, however, the possibility of an almost pure bino dark matter with a mass of up to a few hundreds of $\mathrm{GeV}$ can be viable $[8,9]$. In such scenarios, binos can annihilate into vector-like fermions through the $t$-channel exchange of the corresponding sfermion as illustrated in Fig. 1. In the QUE and QDEE models, these annihilation channels can constitute an efficient-enough mechanism for depleting binos in the early Universe due to the interplay of two effects. First, binos can annihilate into vector-like weaksinglet leptons $E$ carrying hypercharge $Y_{E}=1$. Since the sfermion-mediated annihilation cross section of binos into fermions scales as $Y^{4}$, this annihilation channel is particularly enhanced. Second, although $S U(2)_{L}$ singlet fermions also exist in the MSSM, the corresponding annihilation cross section is suppressed by the masses of the light SM fermions. The vector-like fermions present in MSSM extensions, on the other hand, are (necessarily) much heavier, hence this suppression is no longer present. It is the interplay of these two factors that renders heavier bino dark matter a viable option in the QUE and QDEE models, making it possible to achieve masses as high as $\sim 450 \mathrm{GeV}$ in the former case and $\sim 600 \mathrm{GeV}$ in the latter $[8,9]$. In the LND model, however, the situation is slightly different. Although, in this model too the binos can annihilate into heavy electron (and neutrino) pairs through the $t$-channel exchange of the corresponding sfermions, now the new leptons belong to an $S U(2)_{L}$ doublet with an hypercharge $Y_{L}=1 / 2$. This implies that, all other parameters being identical, the bino annihilation cross section is suppressed by a factor $1 / 16$ relatively to the QUE and QDEE models. We thus expect the phenomenology of neutralino dark matter to be more similar to the MSSM one than that of the other two GUT-inspired MSSM extensions with vector-like fermions.

In Fig. 2 we present the neutralino relic abundance as a function of the dark matter mass, highlighting in different colors the contribution of these novel annihilation channels

\footnotetext{
${ }^{2}$ Natural in the sense of how rapidly the predicted dark matter abundance changes with small variations of the model parameters [50-52].
} 


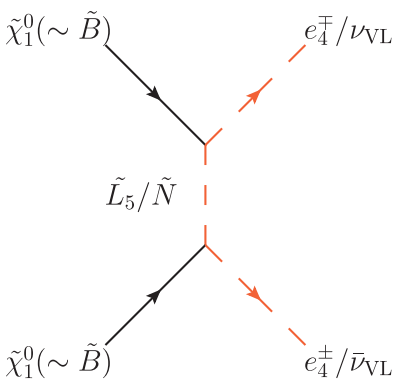

FIG. 1. Representative dark matter annihilation diagram into vector-like fermions.

to the total dark matter annihilation cross section. In the left panel we show all the scenarios probed in our scanning procedure that respect the constraints described in Sec. III B, whereas in the right panel we exclude scenarios in which dark matter depletion is dominated by funnels $(Z / h / H / A)$ or co-annihilations. Several comments are in order.

First, we recover the well-known result that sub-TeV Higgsinos and winos lie below the Planck region (with $\Omega h^{2} \sim 0.12$ [54]), i.e., the predicted relic density is smaller by one or two orders of magnitude than the observed one. This is illustrated on the bottom of the left subfigure as a line-like accumulation of scenarios. In contrast, almost pure bino dark matter that does not annihilate into vector-like fermions can be either overabundant or under-abundant, depending on whether or not co-annihilations and funnels are efficient in depleting DM. The blue parameter space points for which the Planck measurements are exactly met correspond to scenarios of bino dark matter either annihilating through a quasi on-shell $Z / h / H$ or $A$ boson, or coannihilating with MSSM sparticles. Such configurations are also present in the MSSM. The novel feature appearing in the LND model are the red points, which correspond exactly to situations in which binos annihilate into vectorlike leptons. While coannihilations with the corresponding sfermions are also possible, they are not necessary to reproduce the Planck measurements.

Second, we observe the existence of a lower bound in the predicted dark matter abundance for binos annihilating exclusively into vector-like leptons. This limit is due to the fact that the interactions involved in annihilation diagrams such as the one depicted in Fig. 1 result from gauge couplings, which implies that their magnitude is essentially fixed. Then, in the absence of additional annihilation processes, these interactions can be efficient only up to a certain point in depleting dark matter, which corresponds to the observed lower bound in $\Omega h^{2}$. This lower limit scales roughly as the squared bino mass, which is a consequence of the fact that, for large enough dark matter masses, $\langle\sigma v\rangle$ is roughly proportional to the inverse square of the dark matter mass, a dependence which reflects upon the predicted relic density. The situation is fairly similar to the scaling of the wino and/or Higgsino abundance as a function of the neutralino mass.

Third, the rather sharp cutoff observed in the red points around a mass of $100 \mathrm{GeV}$ is simply due to the fact that the vector-like leptons (and, in particular, the heavy electrons) cannot be lighter than about $100 \mathrm{GeV}$, because of the experimental constraints on their mass discussed in Sec. III B.

So, as anticipated, the neutralino dark matter phenomenology we recover is fairly similar to the MSSM one. Due to the hypercharge suppression of processes such as the one depicted in Fig. 1, annihilation into vector-like fermions is not as efficient in the LND model as in the QUE and QDEE ones. It is, hence, not possible to reach bino masses larger than $\sim 200 \mathrm{GeV}$ while imposing all existing experimental constraints and explaining the observed dark matter density

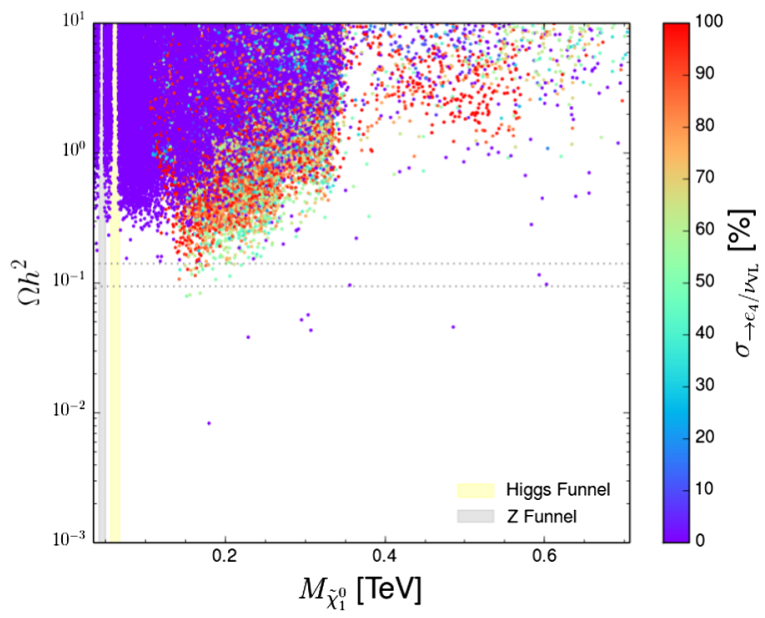

FIG. 2. Relic abundance of neutralino dark matter in the LND model as a function of the dark matter mass, with all known funnels and coannihilation channels (left) or without those channels (right). The different colors correspond to the relative importance of the contributions from annihilations into vector-like fermions to the total dark matter annihilation cross section (blue to red for increasing contribution). The horizontal dashed lines indicate the region favored by Planck data [54]. 

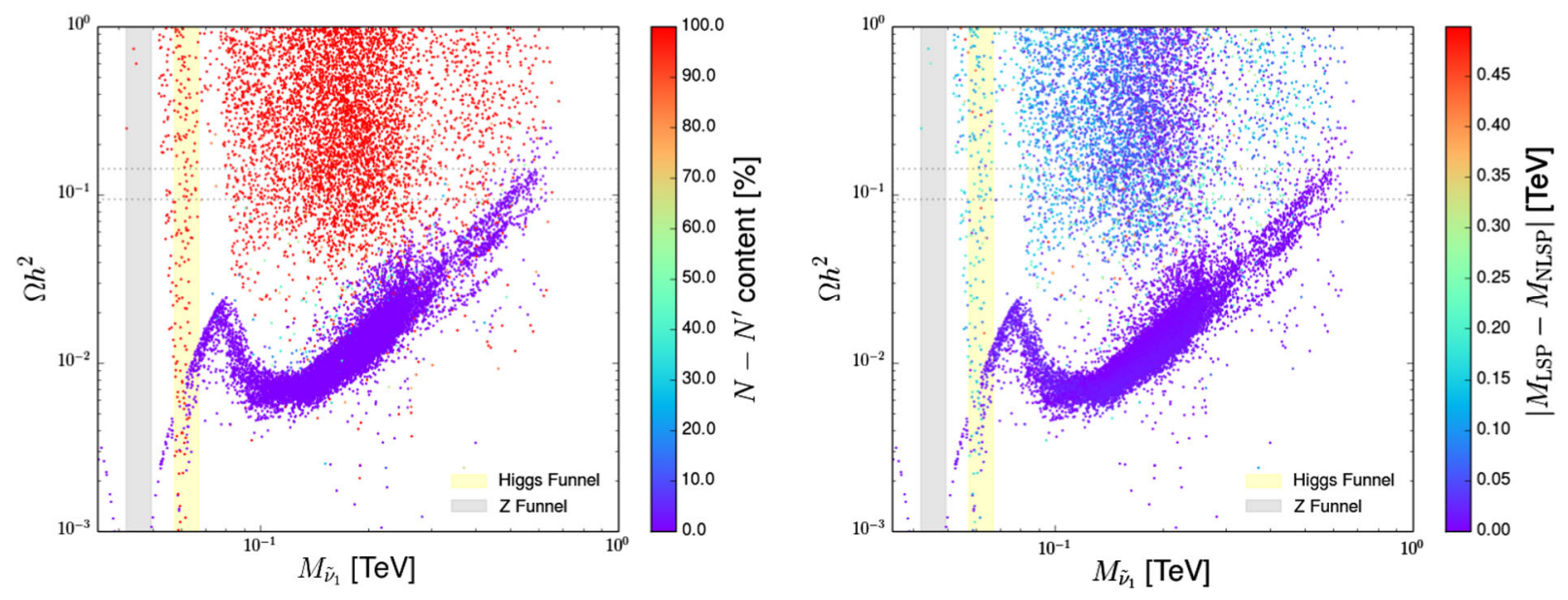

FIG. 3. Sneutrino relic abundance as a function of its mass. In the left panel we highlight in blue the parameter space points for which the lightest sneutrino is dominated by a doublet component, whereas red points are essentially singlet-like. In the right panel, we indicate which of the parameter space points are characterized by large (red) or small (blue) mass splittings between the lightest sneutrino and the NLSP.

in the Universe. Although the dark matter annihilation channels might differ drastically, the accessible masses are at the end comparable to those that would be obtained in the MSSM. However, as we will see in Sec. V, the existence of the vector-like (s)fermions can give rise to interesting, novel phenomenological signatures at the LHC and provide additional handles for collider dark matter searches.

\section{B. Sneutrino dark matter}

As already explained in Sec. II, LND sneutrinos can be a random admixture of the MSSM (SU(2) doublet) lefthanded sneutrinos and the vector-like left- and right-handed $S U(2)$ doublet or singlet ones. A first finding from our parameter space scan is that, as expected (cf. e.g., Ref. [3]), mostly doublet-like sneutrino dark matter can be perfectly compatible with the requirement to reproduce the observed dark matter abundance in the Universe, but is excluded by direct detection experiments due to the strong coupling to the $Z$ boson. This is a well-known feature in the MSSM which persists in the LND model. In order to illustrate it, in the left panel of Fig. 3 we show the sneutrino relic abundance as a function of its mass, highlighting in different colors (red to blue) the increasing doublet fraction. For simplicity, we ignore scenarios with MSSM-like sneutrinos. We observe that mostly doublet-like scenarios (blue points) can satisfy the Planck constraint for sneutrino masses around $600-800 \mathrm{GeV}$, a range which is comparable to the usual MSSM sneutrino dark matter scenario [3]. These scenarios are, nonetheless, found to be in severe conflict with direct detection constraints.

In principle, the presence of additional light leptonic doublets could also provide the necessary contributions to tame down the discrepancy between the measured and predicted values of the anomalous magnetic moment of the muon, despite the fact that mixing is only allowed with the third generation of SM fermions [55]. This appealing option turns out to be strongly disfavored by cosmology, so that one ends up with a situation similar to the MSSM one. From now on, we will not analyze further doublet-like sneutrino DM candidates.

Singlet-like scenarios (red points), on the other hand, offer much more freedom both from the viewpoint of the Planck-allowed sneutrino masses and as far as direct detection constraints are concerned. The abundance of singlet-like scenarios is determined through the interplay of several dark matter depletion processes including direct annihilations into Higgs boson pairs, annihilations through quasi-on-shell $s$-channel scalars and sfermion exchange, and coannihilations. The impact of the latter is in particular illustrated in the right panel of Fig. 3, in which we highlight in different colors (red to blue) scenarios with decreasing mass splitting between the lightest sneutrino and the NLSP and which indicate increasing coannihilation contributions. The observed relic abundance in the Universe can be reproduced for a large range of mass splittings, which implies that sneutrinos can be a cosmologically viable option with or without coannihilations.

The impact of the DM direct and indirect detection constraints for sneutrino dark matter on the LND model is shown in Fig. 4. In the left panel we present the DM-proton spin-independent scattering cross section against the sneutrino mass and compare it with the latest exclusion bounds from LUX [56,57] (red-dashed line) and XENON1T [58] (black-dashed line and shaded $3 \sigma$ band). In order to account for the possibility of sneutrinos comprising only a subleading dark matter component, the scattering cross section has been rescaled according to the predicted dark matter abundance for each scenario, which we assume to scale identically to its present-day local density, an we ignore configurations yielding over-abundant dark matter. In the 

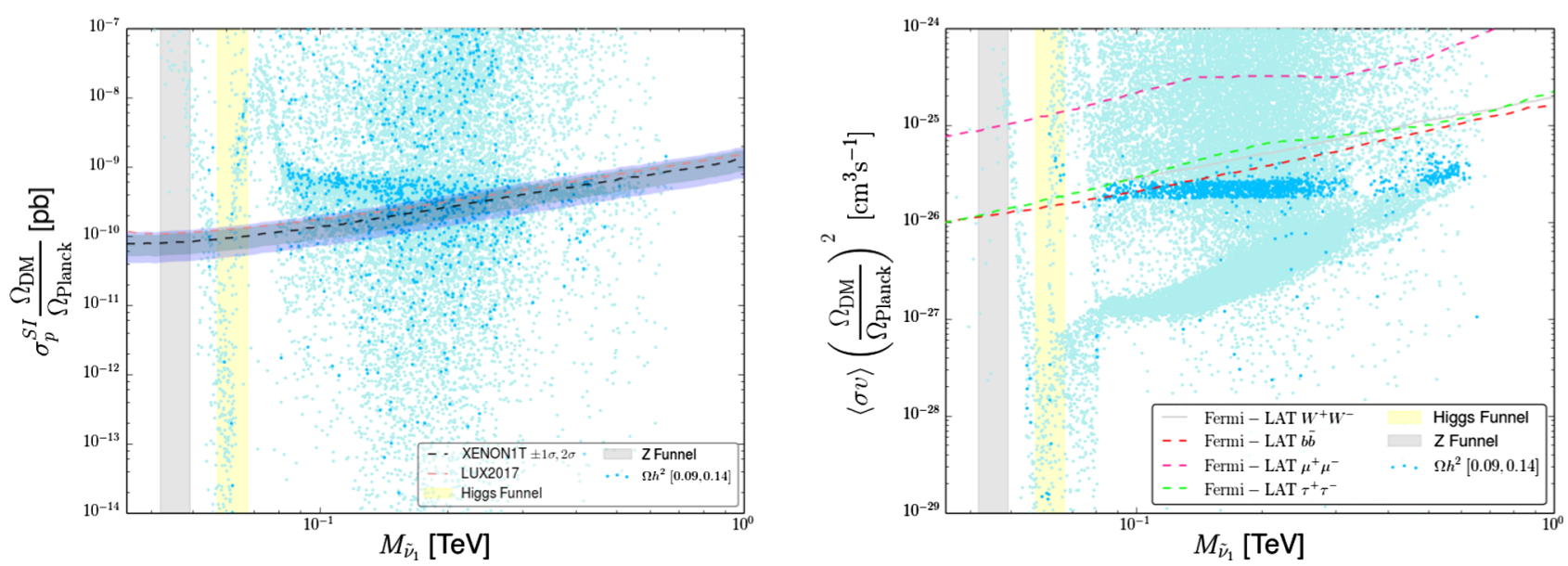

FIG. 4. Direct (left panel) and indirect (right panel) detection constraints on sneutrino dark matter in the LND model as a function of the dark matter mass. In both cases we highlight, in darker blue, the parameter space points for which the two-sided Planck constraint can be satisfied.

right panel we instead show the predicted zero-velocity thermally averaged self-annihilation cross section and compare it with the latest bounds from the Fermi satellite mission [59] for different annihilation channels. Although the exact nature of the annihilation products may vary substantially, the constraints for hadronic (quarks/gauge bosons) channels tend to correspond more to the actual constraints on our scenarios. The self-annihilation cross section has in this case been rescaled by $\Omega_{\text {pred }}^{2} / \Omega_{\text {Planck}}^{2}$, again in order to account for the possibility of underabundant scenarios. In both panels, the parameter space points satisfying both the upper and the lower Planck bound are highlighted in darker blue and we omit points for which dark matter would be over-abundant.

As we can observe from the left panel of Fig. 4, the parameter space points for which $M_{\tilde{\nu}_{1}} \sim M_{h} / 2$ and which satisfy the relic density constraint lie within a band that exhibits a rather standard "funnel"-like behavior. Below this mass value, efficient annihilation of singlet-like sneutrinos into light SM fermions requires rather large couplings to the standard model particles, especially the Higgs boson, which in turn implies that the corresponding scenarios are characterized by relatively large spinindependent scattering cross sections. This brings them in conflict with the recent LUX and XENON1T constraints, which are only satisfied if the sneutrinos annihilate through a quasiresonant Higgs boson in the early Universe. Once the $W$-boson mass threshold is crossed, we observe another abrupt drop in $\sigma_{p}^{S I}$ since for $M_{\tilde{\nu}_{1}}>M_{W}$ the sneutrinos can annihilate directly into a pair of $W$ (and, eventually, $Z$ ) bosons. Still, direct detection constraints exclude most of the parameter space lying within this band for sneutrino masses roughly up to $200 \mathrm{GeV}$. For larger masses, the present-day sensitivity of direct detection experiments is no longer sufficient to exclude these scenarios.

Essentially the same structure is observed in the right panel of Fig. 4, without the sudden drop around $M_{W}$ since the total dark matter self-annihilation cross section remains roughly constant in order to satisfy the Planck bounds. Besides, the occasional scattered points that lie outside the main dark blue band correspond to scenarios with large coannihilation contributions or to cases in which dark matter annihilates predominantly into Higgs boson pairs or vector-like leptons. The larger spread of the dark blue points in the left panel of the figure with respect to the right one is due to the fact that, with the exception of coannihilation, indirect detection probes the same processes that determine the dark matter abundance in the Universe. For instance, if dark matter annihilates predominantly into Higgs boson pairs, its direct detection prospects are rather modest whereas its indirect detection perspectives are almost identical to scenarios with a $W W$ final state, since the final annihilation products are similar and the total thermally averaged self-annihilation cross section is roughly the canonical one.

The DM detection perspectives of the model are hence good, since most of the parameter space that has not yet been excluded by direct or indirect detection lies within a factor of a few from current bounds. We can expect that at least the most "canonical" scenarios will be probed with in the next decade or so. However, LND sneutrino dark matter candidates behave rather similarly to usual sneutrino dark matter in the MSSM augmented with a right-handed neutrino chiral supermultiplet. Most of the features discussed here are present in this scenario too, with the most important differences coming from the existence of a few additional annihilation channels (the contribution of which we have, however, found to be rather modest) and the presence of a handful of additional coannihilation channels. Both of these features do not alter the global picture of sneutrino dark matter with respect to more conventional scenarios. Despite this, we should remind that given the current experimental constraints, essentially all neutralino dark matter scenarios necessitate a $\mu$ parameter that lies at 
the $\mathrm{TeV}$ scale or above. As it has been pointed out [50-52], since the $\mu$ parameter is related to the $Z$-boson mass already at tree-level, this introduces high levels of fine-tuning pushing the theory towards unnatural territories. In sneutrino dark matter scenarios such as the one we just studied, the $\mu$ parameter is decoupled from the dark matter mass and can be fixed much closer to the $Z$-boson mass as required by naturalness. In this respect, the presence of a second dark matter candidate in the form of the (mostly singletlike) sneutrino in the LND model constitutes of an interesting novelty both with respect to the MSSM and to the QUE and QDEE setups.

\section{PROSPECTS AT THE HL-LHC}

The results presented in the previous sections show that there exist LND configurations compatible with cosmological constraints as well as with flavor, Higgs and lowenergy physics observables. We therefore single out several representative benchmark scenarios to study the corresponding LHC phenomenology in more depth, and turn our focus on setups featuring substantial cross sections for vector-like (s)fermion production at the LHC connected to potential novel LHC signatures worthy of investigation. Even though the LND model has some semblance with the MSSM, it exhibits differences due to the existence of additional vector-like leptons and down-type quark. Owing to flavor physics constraints, the vector-like down quark has to be massive and its coupling to the SM quarks has to be small, which reduces its corresponding LHC production cross section significantly. Typical LND signals therefore involve leptonic and often cleaner final states.
We concentrate on vector-like $\tau^{\prime}$ production, in which each extra lepton dominantly decays into a neutral SM-like Higgs-boson $h$ or Z-boson and a tau lepton,

$$
p p \rightarrow \tau^{\prime} \bar{\tau}^{\prime} \rightarrow \tau \bar{\tau} X X \quad \text { with } \quad X=h, Z \text {. }
$$

After accounting for $h$ and Z-boson decays, this process could give rise to a copious production of multileptonic events with small SM backgrounds. We analyse the four benchmark points defined in Table III, that leads to the production of events containing four first and second generation leptons $(e$ or $\mu)$ at the LHC. The tables include the 20 parameters relevant for collider physics, the most relevant ones being $\varepsilon_{N, 3}, k_{N}, \kappa_{L, 3}, h_{N}$ and $\varepsilon_{E}$ as the considered signal involves vector-like $\tau^{\prime}$ states. The corresponding particle spectra are presented in Table IV.

As soon as all branching ratios are properly included, final states containing four leptons $(e$ or $\mu$ ), at least one hadronic tau and no $b$-tagged jets could yield the largest signal sensitivity, highlighted in particular by a low associated background. We make use of MG5_AMC@NLO (version 2.6.1) [42] to generate leading-order hard-scattering events for both the signal for the four considered benchmarks, and for the different components of the SM background, for proton-proton collisions at a center-of-mass energy of $14 \mathrm{TeV}$. We generate events for diboson production (including off-shell effects, once accounting for weak boson leptonic decays), as well as for the subdominant $t \bar{t} h, t \bar{t} Z$ and $t \bar{t} W W$ background contributions. We have additionally verified that triboson background contributions were negligible. In our simulations, we rely on the four-flavor number scheme, making use of the leading-order set of NNPDF2.3

TABLE III. Parameters defining our four representative LND benchmark scenarios BP1-BP4. The sign of the $\mu$ parameter has been taken positive in all cases.

\begin{tabular}{lcccccccc}
\hline \hline & $\mu_{L}[\mathrm{GeV}]$ & $\tan \beta$ & $\varepsilon_{N, 3}$ & $k_{N}$ & $M_{0}[\mathrm{TeV}]$ & $M_{1}[\mathrm{GeV}]$ & $B_{0}[\mathrm{GeV}]$ & $\mu_{D}[\mathrm{TeV}]$ \\
\hline BP1 & 144.9 & 41.6 & -0.045 & 0.013 & 2.2 & 160.8 & 584.64 & 7.22 \\
BP2 & 128.9 & 42.6 & -0.06 & -0.18 & 1.57 & 168.25 & 481.79 & 5.47 \\
BP3 & 132.42 & 40.52 & -0.049 & -0.13 & 1.65 & 156.39 & 452.09 & 6.03 \\
BP4 & 162.96 & 25.36 & -0.035 & 0.0888 & 1.05 & 206.24 & 1306.38 & 4.12 \\
\hline \hline & $M_{2}[\mathrm{TeV}]$ & $\kappa_{L, 3}[\mathrm{GeV}]$ & $h_{N}$ & $\varepsilon_{E}$ & $A_{0}[\mathrm{GeV}]$ & $m_{L^{\prime}, 3}^{2}\left[\mathrm{TeV}^{2}\right]$ & $m_{D^{\prime}, 3}^{2}\left[\mathrm{TeV}^{2}\right]$ & $m_{L, 3}^{2}\left[\mathrm{TeV}^{2}\right]$ \\
\hline BP1 & 1.5 & -11.9 & -0.038 & -0.29 & 95.26 & 0.15 & 1.76 & $4.78 \times 10^{-3}$ \\
BP2 & 1.05 & 1.28 & $-6.8 \times 10^{-3}$ & -0.16 & -743.38 & $2.91 \times 10^{-3}$ & 1.29 & $6.86 \times 10^{-3}$ \\
BP3 & 1.01 & 1.16 & $-4.9 \times 10^{-3}$ & -0.16 & -516.61 & $2.09 \times 10^{-3}$ & 1.55 & $7.58 \times 10^{-3}$ \\
BP4 & 0.45 & 15.74 & -0.106 & $2.47 \times 10^{-3}$ & $5.51 \times 10^{-4}$ & 0.25 & 23.68 & 0.20 \\
\hline \hline & $m_{N, 3}^{2}\left[\mathrm{TeV}^{2}\right]$ & $m_{N^{\prime}, 3}^{2}\left[\mathrm{TeV}^{2}\right]$ & $\mu_{N}[\mathrm{GeV}]$ & $m_{D, 3}^{2}\left[\mathrm{TeV}^{2}\right]$ & $\kappa_{D}[\mathrm{GeV}]$ & $\varepsilon_{D}$ & $m_{L_{5}, L, 3}^{2}\left[\mathrm{TeV}^{2}\right]$ & $m_{D_{5}, D, 3}^{2}\left[\mathrm{TeV}^{2}\right]$ \\
\hline BP1 & 0.32 & $2.28 \times 10^{-2}$ & 748.84 & 10.59 & 0.0 & $-2.38 \times 10^{-4}$ & $-9.16 \times 10^{-5}$ & $7.89 \times 10^{-5}$ \\
BP2 & $1.32 \times 10^{-3}$ & $1.11 \times 10^{-3}$ & 984.28 & $2.46 \times 10^{-3}$ & 0.0 & $-9.47 \times 10^{-4}$ & $-4.41 \times 10^{-5}$ & $-2.91 \times 10^{-5}$ \\
BP3 & $1.24 \times 10^{-3}$ & $8.29 \times 10^{-4}$ & 975.48 & $3.31 \times 10^{-3}$ & 0.0 & $-1.08 \times 10^{-3}$ & $-4.50 \times 10^{-5}$ & $-2.69 \times 10^{-5}$ \\
BP4 & 0.29 & 0.12 & 1499.71 & 14.89 & 0.0 & $-3.06 \times 10^{-4}$ & $-9.81 \times 10^{-5}$ & $3.86 \times 10^{-5}$ \\
\hline \hline
\end{tabular}


TABLE IV. Masses of the particles lighter than 2.5 TeV for our four representative LND benchmark scenarios BP1-BP4.

\begin{tabular}{lccccccc}
\hline \hline & $M_{\tau^{\prime}}[\mathrm{GeV}]$ & $M_{\tilde{\chi}_{1}^{0}}[\mathrm{GeV}]$ & $M_{\nu_{1}^{\prime}}[\mathrm{GeV}]$ & $M_{\nu_{2}^{\prime}}[\mathrm{GeV}]$ & $M_{b^{\prime}}[\mathrm{TeV}]$ & $M_{\tilde{\chi}_{2}^{0}} \cong M_{\tilde{\chi}_{1}^{ \pm}}[\mathrm{TeV}]$ & $M_{\tilde{e}_{1}}[\mathrm{GeV}]$ \\
\hline BP1 & 150.87 & 157.48 & 146.71 & 748.95 & 7.36 & 1.53 & 175.73 \\
BP2 & 133.98 & 164.91 & 130.22 & 985.49 & 5.64 & 1.09 & 188.66 \\
BP3 & 137.41 & 153.33 & 133.68 & 976.16 & 6.16 & 1.05 & 175.47 \\
BP4 & 168.72 & 202.55 & 164.21 & 1500.53 & 4.28 & 0.48 & 221.56 \\
\hline \hline & $M_{\tilde{e}_{2}}[\mathrm{GeV}]$ & $M_{\tilde{\nu}_{1}}[\mathrm{GeV}]$ & $M_{\tilde{\nu}_{2}}[\mathrm{GeV}]$ & $M_{\tilde{\nu}_{3}}[\mathrm{GeV}]$ & $M_{\tilde{\nu}_{4}}[\mathrm{TeV}]$ & $M_{\tilde{\nu}_{5}}[\mathrm{TeV}]$ & $M_{\tilde{\nu}_{6}}[\mathrm{TeV}]$ \\
\hline BP1 & 516.31 & 169.23 & 520.67 & 633.22 & 1.02 & 1.47 & 1.81 \\
BP2 & 401.71 & 193.82 & 401.03 & 491.87 & 1.31 & 1.78 & 2.39 \\
BP3 & 399.22 & 182.70 & 396.97 & 459.45 & 1.31 & 1.38 & 1.99 \\
BP4 & 691.04 & 219.54 & 691.25 & 1537.79 & 1.59 & 1.80 & 2.39 \\
\hline \hline
\end{tabular}

FIG. 5. Distributions in the $H_{T}, M_{\text {eff }}$ and $M_{4 \ell}$ observables for the BP1 benchmark scenario and the dominant contributions to the SM background, once the preselection cuts of Eq. (5.3) have been imposed. The normalization is arbitrary.

parton densities [60]. We include taus when enforcing weak boson leptonic decays, and allow for the presence of up to two extra partons in the final state. The multipartonic contributions are then merged following the MLM prescription [61]. Parton showering and hadronization are performed within the PYTHIA8 (version 8.230) framework [62] and we simulate the response of the detector by means of DELPHES 3 (version 3.4.1) [63]. We modify slightly the default CMS detector parametrization that relies on the FASTJET program (version 3.2.1) [64] for jet reconstruction on the basis of the anti- $k_{T}$ algorithm [65], with a radius parameter set to $R=0.5$. Our modifications imply a tau-tagging efficiency fixed to $60 \%$, for a mistagging rate of a light-jet as a hadronic tau set to $1 \%$ (this configuration matches average performances after the object selection enforced below). In contrast, we consider standard $b$-tagging performance as implemented in the default CMS parametrization [66].

We define the relevant reconstructed object candidates by imposing transverse momentum $\left(p_{T}\right)$ and pseudorapidity $(\eta)$ conditions on the leptons $(\ell=e, \mu)$, hadronic taus $\left(\tau_{h}\right)$ and light and $b$-tagged jets $(j$ and $b)$,

$p_{T}^{\ell} \geq 10 \mathrm{GeV}$ and $\left|\eta^{\ell}\right|<2.5, \quad p_{T}^{\tau_{h}} \geq 20 \mathrm{GeV}$ and

$\left|\eta^{\tau}\right|<2.5, \quad p_{T}^{j, b} \geq 30 \mathrm{GeV}, \quad\left|\eta^{j}\right|<4.5 \quad$ and

$\left|\eta^{b}\right|<2.5$.
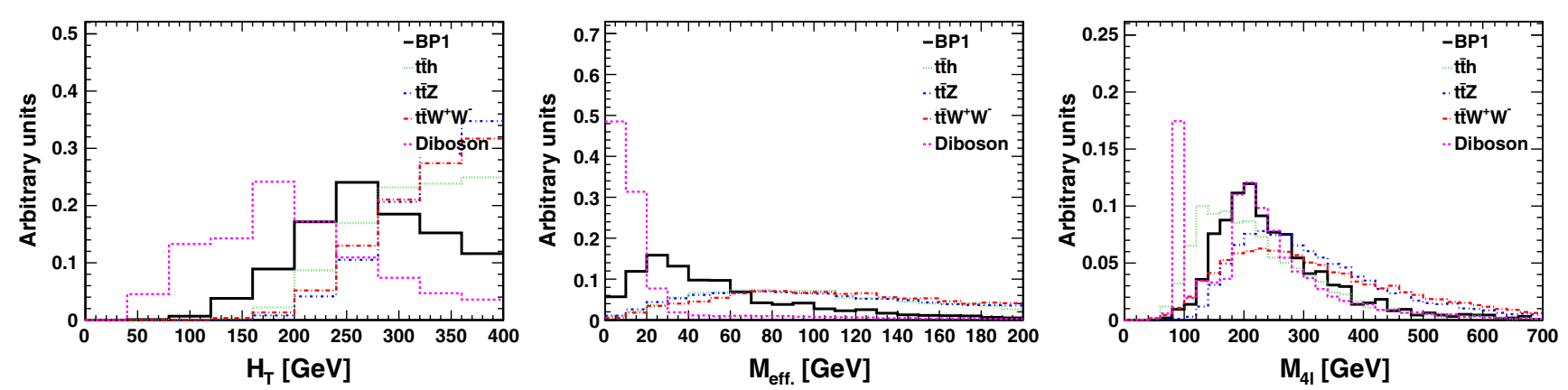

We moreover require lepton isolation by imposing that the total hadronic activity within a cone of radius $\Delta R=0.5$ around any electron (muon) is smaller than 12\% (25\%) of the lepton $p_{T}$, and that all reconstructed leptons are separated from each other by an angular distance, in the transverse plane, of at least $R=0.5$. We then preselect events by constraining the number of reconstructed finalstate electrons and muons $\left(N^{\ell}\right)$, hadronic taus $\left(N^{\tau_{h}}\right)$ and $b$-tagged jets $\left(N^{b}\right)$, to be

$$
N^{\ell} \geq 4, \quad N^{b}=0 \quad \text { and } \quad N^{\tau_{h}} \geq 1 .
$$

We then investigated a large set of observables and found that the most discriminatory ones are the total transverse activity $H_{T}$ (the scalar sum of the $p_{T}$ of all reconstructed visible objects), a modified version of the effective mass $M_{\text {eff }}$ (the scalar sum of the $p_{T}$ of all jets and the missing transverse energy $E_{T}$ ), and the invariant mass $M_{4 \ell}$ of the system made of the four hardest leptons. The distributions in these variables are shown in Fig. 5 for both the different background contributions and the illustrative BP1 benchmark scenario. Upon scrutinizing these variables, we select events for which

$$
H_{T}>250 \mathrm{GeV}, \quad M_{\text {eff }}>30 \mathrm{GeV} \text { and }
$$$$
M_{4 \ell}>200 \mathrm{GeV} \text {. }
$$ 
TABLE V. Impact of our event selection strategy on the SM background and the four considered benchmark scenarios. For each cut, we provide the expected number of surviving events for $\mathcal{L}=3 \mathrm{ab}^{-1}$ of LHC collisions at a center-of-mass energy of $14 \mathrm{TeV}$. We also quote the corresponding significances $s$ and $Z_{A}$ defined in Eq. (5.5), including a $20 \%$ systematic uncertainty on the background. We additionally indicate, in parentheses, the significances for a lower luminosity of $300 \mathrm{fb}^{-1}$.

\begin{tabular}{lcccccc}
\hline \hline Step & Requirements & Background & BP1 & BP2 & BP3 & BP4 \\
\hline 0 & Initial & $5.3 \times 10^{9}$ & $1.4 \times 10^{8}$ & $2.0 \times 10^{8}$ & $2.1 \times 10^{8}$ & $1.7 \times 10^{8}$ \\
1 & Preselection & 149 & 241 & 336 & 336 & 282 \\
2 & $H_{T}>250 \mathrm{GeV}$ & 101 & 183 & 240 & 247 & 236 \\
3 & $M_{\mathrm{eff}}>30 \mathrm{GeV}$ & 39 & 117 & 120 & 125 & 156 \\
4 & $M_{4 \ell}>200 \mathrm{GeV}$ & 34 & 90 & 95 & 100 & 118 \\
& $3 \mathrm{ab}^{-1}\left(300 \mathrm{fb}^{-1}\right)$ & $S$ & $10.06 \sigma(4.59 \sigma)$ & $10.57 \sigma(4.82 \sigma)$ & $11.13 \sigma(5.08 \sigma)$ & $13.16 \sigma(6.00 \sigma)$ \\
& & $Z_{A}$ & $6.70 \sigma(3.39 \sigma)$ & $6.94 \sigma(3.53 \sigma)$ & $7.22 \sigma(3.68 \sigma)$ & $8.14 \sigma(4.19 \sigma)$ \\
\hline \hline
\end{tabular}

Whilst further optimization is possible, these choices allow for a good enough background rejection in the context of the four considered benchmark selections, as illustrated by the detailed cutflow charts shown in Table V for $3 \mathrm{ab}^{-1}$ of LHC collisions at $14 \mathrm{TeV}$.

We evaluate the sensitivity of the high-luminosity LHC to our different benchmark scenarios by computing two significance indicators $s$ and $Z_{A}$ defined by [67]

$$
s=\frac{S}{\sqrt{B+\sigma_{B}^{2}}} \quad \text { and } \quad Z_{A}=\sqrt{2\left((S+B) \ln \left[\frac{(S+B)\left(S+\sigma_{B}^{2}\right)}{B^{2}+(S+B) \sigma_{B}^{2}}\right]-\frac{B^{2}}{\sigma_{B}^{2}} \ln \left[1+\frac{\sigma_{B}^{2} S}{B\left(B+\sigma_{B}^{2}\right)}\right]\right)},
$$

with $S$ and $B$ being respectively the total number of signal and background events surviving the selection. Our results assume a $20 \%$ systematic uncertainty on the SM background, $\sigma_{B}=0.2 \times B$. In Table $\mathrm{V}$ we compare the expectation for both $300 \mathrm{fb}^{-1}$ and $3 \mathrm{ab}^{-1}$ of LHC collisions. The discovery prospects are in all cases very promising, so that the presence of the vector-like leptons offers good handles on LND models. It will, however, be challenging to conclude about the realization of the model in nature without getting a grip on the supersymmetric part of the spectrum with the LHC alone.

This could for instance be achieved by investigating the impact of the searches for supersymmetry through its monojet and multijet plus missing energy signatures [68-71]. In all cosmologically favored LND setups analyzed in Sec. IV, such signals arise from the pair production of squarks and/or gluinos. However, the lightest squarks have generally masses of about $2 \mathrm{TeV}$ or more, so that the corresponding cross sections are negligibly small, especially after imposing the presence of at least one very hard jet in the final state. Exceptional scenarios nevertheless exist, in which lighter colored superpartners are featured. In this case, the relevant cross sections are of $\mathcal{O}(1) \mathrm{fb}$, which is too small to yield any hope of observing a hint for the signal. We have, in addition, evaluated the cross sections associated with electroweakino pair production. For all scenarios favored by cosmological data, they reach at most $0.1 \mathrm{fb}$, when the branching-ratiofavored hadronic final states are considered. The direct observation of a supersymmetric signal at the LHC will, hence, be very unlikely. Thankfully, as demonstrated in this work, the presence of vector-like leptons in the LND model provides additional observational handles which are complementary to cosmological and astrophysical probes.

Our collider analysis focused on decays into vector-like $\tau^{\prime}$ leptons, yielding signals with four leptons (electrons or muons), one hadronic tau and no $b$-jets. This constitutes a rather unique signature that is neither probed by multilepton analyses, which target final states with three or more leptons but no taus [72], nor by conventional searches for vector-like leptons, which include either three or more light leptons and no taus, or two light leptons and a single tau, as in Ref. [73]. Perhaps the closest experimental study to our proposal is the ATLAS analysis of Ref. [74]. However, the information provided in their Tables 4 and 6 indicate that not a single one of their signal region matches ours. Hopefully, further analyses at the LHC will allow for the investigation of a wider range of parameter space and probe additional multilepton signals.

\section{SUMMARY AND CONCLUSIONS}

In this work we presented an analysis of an extension of the MSSM along lines suggested by supersymmetric grand unification. The minimal superfield content of the MSSM is enlarged by the addition of a complete $\mathbf{5} \oplus \overline{\mathbf{5}}$ representation of $S U(5)$, which leads to vector-like pairs of down-type quark and lepton supermultiplets after 
the breaking of the grand-unified symmetry. We moreover include a vector-like pair of singlet neutrino supermultiplets. The model then provides, in addition to the lightest neutralino, a new potential candidate for dark matter compared with other GUT-inspired supersymmetric models with vector-like fermions, namely the scalar superpartner of the singlet vector-like neutrino (the latter becomes a sterile neutrino).

We set up the model allowing mixing of the new vectorlike states with the third generation of down-type quarks and leptons only (to avoid unwanted flavor-changing effects), and investigated its consequences by scanning over the free parameters over a wide range. The parameter space is then restricted by imposing collider mass limits, the compatibility with a complete set of Higgs-sectorrelated measurements, and constraints originating from electroweak precision tests, lepton flavor violation and $B$-physics. We first investigated the dark matter candidates featured by the model and imposed restrictions from the requirement to reproduce the observed dark matter abundance in the Universe and the absence of a signal in direct and indirect dark matter detection experiments.

The neutralino DM candidates turn out to have fairly similar properties as in the MSSM. In particular, in this model the bino-(vector-like) electron-selectron coupling is a gauge coupling, and thus proportional to the hypercharge $Y$. The bino-like neutralino annihilation cross section into vector-like fermions is, then, proportional to $Y^{4}$, and since the vector-like (s)fermions that are charged under $U(1)_{Y}$ belong to $S U(2)_{L}$ doublets they carry a hypercharge of $1 / 2$. This, in turn, implies that the overall bino annihilation cross section is suppressed in comparison to models containing vector-like $S U(2)_{L}$ singlets. As we showed explicitly, this suppression hinders these novel (with respect to the MSSM) annihilation channels from providing an efficient mechanism for dark matter depletion in the early Universe and deprives heavier binos of the possibility to be viable DM candidates.

While sneutrinos can in principle be an arbitrary admixture of the MSSM $\left(S U(2)_{L}\right.$ doublet) left-handed sneutrinos and the vector-like left- and right-handed $S U(2)_{L}$ doublet or singlet ones, and while the mostly doublet-like sneutrino dark matter option is entirely compatible with the requirement to reproduce the observed relic density, direct detection experiments exclude such scenarios. This means that only singlet-like sneutrinos survive, making this scenario difficult to differentiate from other models where the right-handed sneutrino is included on an ad-hoc basis, or is required by the symmetry of the model. However, as we argued, given all current experimental constraints sneutrino dark matter in this scenario can further find motivation from naturalness arguments, which constitutes an interesting twist of the LND model with respect to other minimal GUT-motivated MSSM extensions with vector-like fermions, like the QUE and QDEE models.

Lastly, the model shows some interesting prospects in collider signals. We devised benchmark points with substantial cross sections to vector-like final states, to unravel the corresponding signals that are absent from the MSSM. We concentrated on vector-like $\tau^{\prime}$ production, decaying dominantly into a neutral boson (either the SM-like Higgs boson $h$ or the $Z$ boson) and a tau lepton. We have demonstrated that the pair-production of a pair of vectorlike $\tau^{\prime}$ yields a multilepton signature that could distinguish this model in the future high-luminosity runs of the LHC, for both luminosities of $300 \mathrm{fb}^{-1}$ and $3 \mathrm{ab}^{-1}$. Taken together with the cosmological implications of the model, this signal could provide a way to assess its viability.

\section{ACKNOWLEDGMENTS}

We thank F. Staub and A. Pukhov for their respective help with the SARAH and MicrOMEGAs packages, as well as S. Jain for helpful discussions on the collider front. This research was enabled in part by support provided by the High Performance Computing (HPC) server Guillimin in McGill University (hpc.mcgill.ca) and Compute Canada (computecanada.ca). J. Y. A. and M. F. acknowledges Natural Sciences and Engineering Research Council (NSERC) for partial financial support under Grant No. SAP105354 and J. Y. A. acknowledges the hospitality of the LPTHE, Sorbonne University and INFNLaboratori Nazionali di Frascati where part of this work was completed. The work of B.F. and A. G. is partly supported by French state funds managed by the Agency Nationale de la Recherche (ANR), in the context of the LABEX ILP (ANR-11-INDEX-0004-02, ANR-10-LAB63). S. B. is supported by a Durham Junior Research Fellowship COFUNDed by Durham University and the European Union, under Grant Agreement No. 609412, and acknowledges the hospitality of the CERN Theory Department where part of this work was completed.
[1] J. A. Casas, J. R. Espinosa, and I. Hidalgo, The MSSM fine tuning problem: A way out, J. High Energy Phys. 01 (2004) 008 .
[2] M. Dine, N. Seiberg, and S. Thomas, Higgs physics as a window beyond the MSSM (BMSSM), Phys. Rev. D 76, 095004 (2007). 
[3] C. Arina and N. Fornengo, Sneutrino cold dark matter, a new analysis: Relic abundance and detection rates, J. High Energy Phys. 11 (2007) 029.

[4] U. Ellwanger, C. Hugonie, and A. M. Teixeira, The next-tominimal supersymmetric standard model, Phys. Rep. 496, 1 (2010).

[5] P. Batra, A. Delgado, D. E. Kaplan, and T. M. P. Tait, The Higgs mass bound in gauge extensions of the minimal supersymmetric standard model, J. High Energy Phys. 02 (2004) 043.

[6] K. S. Babu and R. N. Mohapatra, Minimal supersymmetric left-right model, Phys. Lett. B 668, 404 (2008).

[7] S. P. Martin, Extra vector-like matter and the lightest Higgs scalar boson mass in low-energy supersymmetry, Phys. Rev. D 81, 035004 (2010).

[8] M. Abdullah and J. L. Feng, Reviving bino dark matter with vectorlike fourth generation particles, Phys. Rev. D 93, 015006 (2016).

[9] M. Abdullah, J. L. Feng, S. Iwamoto, and B. Lillard, Heavy bino dark matter and collider signals in the MSSM with vectorlike fourth-generation particles, Phys. Rev. D 94, 095018 (2016).

[10] C. Athanassopoulos et al. (LSND Collaboration), Evidence for $\nu_{\mu} \rightarrow \nu_{e}$ Neutrino Oscillations from LSND, Phys. Rev. Lett. 81, 1774 (1998).

[11] A. A. Aguilar-Arevalo et al. (MiniBooNE Collaboration), Improved Search for $\bar{\nu}_{\mu} \rightarrow \bar{\nu}_{e}$ Oscillations in the MiniBooNE Experiment, Phys. Rev. Lett. 110, 161801 (2013).

[12] T. Moroi and Y. Okada, Radiative corrections to Higgs masses in the supersymmetric model with an extra family and antifamily, Mod. Phys. Lett. A 07, 187 (1992).

[13] T. Moroi and Y. Okada, Upper bound of the lightest neutral Higgs mass in extended supersymmetric Standard Models, Phys. Lett. B 295, 73 (1992).

[14] K. S. Babu, I. Gogoladze, and C. Kolda, Perturbative unification and Higgs boson mass bounds, arXiv:hep-ph/ 0410085 .

[15] K. S. Babu, I. Gogoladze, M. U. Rehman, and Q. Shafi, Higgs boson mass, sparticle spectrum and little hierarchy problem in extended MSSM, Phys. Rev. D 78, 055017 (2008).

[16] J. Ellis and K. A. Olive, Supersymmetric dark matter candidates, arXiv:1001.3651.

[17] C. Patrignani et al. (Particle Data Group Collaboration), Review of particle physics, Chin. Phys. C 40, 100001 (2016).

[18] F. Staub, SARAH 4: A tool for (not only SUSY) model builders, Comput. Phys. Commun. 185, 1773 (2014).

[19] W. Porod and F. Staub, SPheno 3.1: Extensions including flavour, $C P$-phases and models beyond the MSSM, Comput. Phys. Commun. 183, 2458 (2012).

[20] Y. Amhis et al. (HFLAV Collaboration), Averages of $b$-hadron, $c$-hadron, and $\tau$-lepton properties as of summer 2016, Eur. Phys. J. C 77, 895 (2017).

[21] D. Asner et al. (Heavy Flavor Averaging Group Collaboration), Averages of $b$-hadron, $c$-hadron, and $\tau$-lepton properties, arXiv:1010.1589.

[22] R. Aaij et al. (LHCb Collaboration), First Evidence for the Decay $B_{s}^{0} \rightarrow \mu^{+} \mu^{-}$, Phys. Rev. Lett. 110, 021801 (2013).
[23] B. Aubert et al. (BABAR Collaboration), Searches for Lepton Flavor Violation in the Decays $\tau \rightarrow e \gamma$ and $\tau \rightarrow \mu \gamma$, Phys. Rev. Lett. 104, 021802 (2010).

[24] Y. Miyazaki et al. (Belle Collaboration), Search for lepton flavor violating $\tau^{-}$decays into $\ell^{-} \eta, \ell^{-} \eta^{\prime}$ and $\ell^{-} \pi^{0}$, Phys. Lett. B 648, 341 (2007).

[25] G. Aad et al. (ATLAS Collaboration), Probing lepton flavour violation via neutrinoless $\tau \rightarrow 3 \mu$ decays with the ATLAS detector, Eur. Phys. J. C 76, 232 (2016).

[26] T. Jubb, M. Kirk, A. Lenz, and G. Tetlalmatzi-Xolocotzi, On the ultimate precision of meson mixing observables, Nucl. Phys. B915, 431 (2017).

[27] G. Aad et al. (ATLAS Collaboration), Search for the lepton flavor violating decay $Z \rightarrow e \mu$ in pp collisions at $\sqrt{s}=$ $8 \mathrm{TeV}$ with the ATLAS detector, Phys. Rev. D 90, 072010 (2014).

[28] V. Khachatryan et al. (CMS Collaboration), Search for lepton flavour violating decays of the Higgs boson to $e \tau$ and $e \mu$ in proton-proton collisions at $\sqrt{s}=8 \mathrm{TeV}$, Phys. Lett. B 763, 472 (2016).

[29] M. Baak, J. Cuth, J. Haller, A. Hoecker, R. Kogler, K. Mönig, M. Schott, and J. Stelzer (Gfitter Group Collaboration), The global electroweak fit at NNLO and prospects for the LHC and ILC, Eur. Phys. J. C 74, 3046 (2014).

[30] D. Eriksson, J. Rathsman, and O. Stal, 2HDMC: TwoHiggs-doublet model calculator physics and manual, Comput. Phys. Commun. 181, 189 (2010).

[31] A. Ilnicka, M. Krawczyk, and T. Robens, Inert doublet model in light of LHC Run I and astrophysical data, Phys. Rev. D 93, 055026 (2016).

[32] P. Bechtle, O. Brein, S. Heinemeyer, G. Weiglein, and K. E. Williams, HiggsBounds: Confronting arbitrary Higgs sectors with exclusion bounds from LEP and the Tevatron, Comput. Phys. Commun. 181, 138 (2010).

[33] P. Bechtle, S. Heinemeyer, O. Stal, T. Stefaniak, and G. Weiglein, HiggsSignals: Confronting arbitrary Higgs sectors with measurements at the Tevatron and the LHC, Eur. Phys. J. C 74, 2711 (2014).

[34] R. V. Harlander, S. Liebler, and H. Mantler, SusHi: A program for the calculation of Higgs production in gluon fusion and bottom-quark annihilation in the Standard Model and the MSSM, Comput. Phys. Commun. 184, 1605 (2013).

[35] V. Khachatryan et al. (CMS Collaboration), Search for new physics with the $\mathbf{M}_{T 2}$ variable in all-jets final states produced in pp collisions at $\sqrt{s}=13 \mathrm{TeV}$, J. High Energy Phys. 10 (2016) 006.

[36] M. Aaboud et al. (ATLAS Collaboration), Search for top squarks in final states with one isolated lepton, jets, and missing transverse momentum in $\sqrt{s}=13 \mathrm{TeV} p p$ collisions with the ATLAS detector, Phys. Rev. D 94, 052009 (2016).

[37] P. Achard et al. (L3 Collaboration), Search for heavy neutral and charged leptons in $e^{+} e^{-}$annihilation at LEP, Phys. Lett. B 517, 75 (2001).

[38] S. Chatrchyan et al. (CMS Collaboration), Observation of a new boson at a mass of $125 \mathrm{GeV}$ with the CMS experiment at the LHC, Phys. Lett. B 716, 30 (2012).

[39] W. K. Hastings, Monte Carlo sampling methods using Markov chains and their applications, Biometrika 57, 97 (1970). 
[40] G. Bélanger, F. Boudjema, A. Pukhov, and A. Semenov, MicrOMEGAs4.1: Two dark matter candidates, Comput. Phys. Commun. 192, 322 (2015).

[41] A. Belyaev, N. D. Christensen, and A. Pukhov, CalcHEP 3.4 for collider physics within and beyond the Standard Model, Comput. Phys. Commun. 184, 1729 (2013).

[42] J. Alwall, R. Frederix, S. Frixione, V. Hirschi, F. Maltoni, O. Mattelaer, H.-S. Shao, T. Stelzer, P. Torrielli, and M. Zaro, The automated computation of tree-level and next-to-leading order differential cross sections, and their matching to parton shower simulations, J. High Energy Phys. 07 (2014) 079.

[43] C. Degrande, C. Duhr, B. Fuks, D. Grellscheid, O. Mattelaer, and T. Reiter, UFO-The universal FeynRules output, Comput. Phys. Commun. 183, 1201 (2012).

[44] A. Buckley, PySLHA: A Pythonic interface to SUSY Les Houches Accord data, Eur. Phys. J. C 75, 467 (2015).

[45] A. Heister et al. (ALEPH Collaboration), Search for charginos nearly mass degenerate with the lightest neutralino in $e^{+} e^{-}$collisions at center-of-mass energies up to 209-GeV, Phys. Lett. B 533, 223 (2002).

[46] J. Abdallah et al. (DELPHI Collaboration), Searches for supersymmetric particles in $e^{+} e^{-}$collisions up to $208-\mathrm{GeV}$ and interpretation of the results within the MSSM, Eur. Phys. J. C 31, 421 (2003).

[47] M. Acciarri et al. (L3 Collaboration), Search for charginos and neutralinos in $e^{+} e^{-}$collisions at $\sqrt{S}=189-\mathrm{GeV}$, Phys. Lett. B 472, 420 (2000).

[48] G. Abbiendi et al. (OPAL Collaboration), Search for chargino and neutralino production at $s^{* *}(1 / 2)=$ 192-GeV to $209 \mathrm{GeV}$ at LEP, Eur. Phys. J. C 35, 1 (2004).

[49] E. Aprile et al. (XENON Collaboration), First Dark Matter Search Results from the XENON1T Experiment, Phys. Rev. Lett. 119, 181301 (2017).

[50] M. Perelstein and B. Shakya, Fine-tuning implications of direct dark matter searches in the MSSM, J. High Energy Phys. 10 (2011) 142.

[51] M. Perelstein and B. Shakya, XENON100 implications for naturalness in the MSSM, NMSSM, and $\lambda$-supersymmetry model, Phys. Rev. D 88, 075003 (2013).

[52] G. Belanger, C. Delaunay, and A. Goudelis, The dark side of electroweak naturalness beyond the MSSM, J. High Energy Phys. 04 (2015) 149.

[53] S. Profumo, T. Stefaniak, and L. Stephenson Haskins, The not-so-well tempered neutralino, Phys. Rev. D 96, 055018 (2017).

[54] P. A. R. Ade et al. (Planck Collaboration), Planck 2015 results. XIII. Cosmological parameters, Astron. Astrophys. 594, A13 (2016).

[55] A. Choudhury, L. Darmé, L. Roszkowski, E. M. Sessolo, and S. Trojanowski, Muon g-2 and related phenomenology in constrained vector-like extensions of the MSSM, J. High Energy Phys. 05 (2017) 072.

[56] D. S. Akerib et al. (LUX Collaboration), Improved Limits on Scattering of Weakly Interacting Massive Particles from Reanalysis of 2013 LUX Data, Phys. Rev. Lett. 116, 161301 (2016).

[57] M. Mitchell, B. Muftakhidinov, T. Winchen, Z. JędrzejewskiSzmek, and The Gitter Badger, Engauge-digitizer: Support for smaller monitors (2016), DOI: 10.5281/zenodo.61108.
[58] E. Aprile et al. (XENON Collaboration), Physics reach of the XENON1T dark matter experiment, J. Cosmol. Astropart. Phys. 04 (2016) 027.

[59] M. Ackermann et al. (Fermi-LAT Collaboration), Searching for Dark Matter Annihilation from Milky Way Dwarf Spheroidal Galaxies with Six Years of Fermi Large Area Telescope Data, Phys. Rev. Lett. 115, 231301 (2015).

[60] R. D. Ball et al., Parton distributions with LHC data, Nucl. Phys. B867, 244 (2013).

[61] M. L. Mangano, M. Moretti, F. Piccinini, and M. Treccani, Matching matrix elements and shower evolution for topquark production in hadronic collisions, J. High Energy Phys. 01 (2007) 013.

[62] T. Sjostrand, S. Mrenna, and P.Z. Skands, A brief introduction to PYTHIA 8.1, Comput. Phys. Commun. 178, 852 (2008).

[63] J. de Favereau, C. Delaere, P. Demin, A. Giammanco, V. Lematre, A. Mertens, and M. Selvaggi (DELPHES 3 Collaboration), DELPHES 3, A modular framework for fast simulation of a generic collider experiment, J. High Energy Phys. 02 (2014) 057.

[64] M. Cacciari, G. P. Salam, and G. Soyez, FastJet user manual, Eur. Phys. J. C 72, 1896 (2012).

[65] M. Cacciari, G. P. Salam, and G. Soyez, The Anti-k(t) jet clustering algorithm, J. High Energy Phys. 04 (2008) 063.

[66] S. Chatrchyan et al. (CMS Collaboration), Identification of $b$-quark jets with the CMS experiment, J. Instrum. 8, P04013 (2013).

[67] G. Cowan, K. Cranmer, E. Gross, and O. Vitells, Asymptotic formulae for likelihood-based tests of new physics, Eur. Phys. J. C 71, 1554 (2011).

[68] M. Aaboud et al. (ATLAS Collaboration), Search for new phenomena in final states with an energetic jet and large missing transverse momentum in $p p$ collisions at $\sqrt{s}=$ $13 \mathrm{TeV}$ using the ATLAS detector, Phys. Rev. D 94, 032005 (2016).

[69] M. Aaboud et al. (ATLAS Collaboration), Search for squarks and gluinos in final states with jets and missing transverse momentum at $\sqrt{s}=13 \mathrm{TeV}$ with the ATLAS detector, Eur. Phys. J. Spec. Top. 76, 392 (2016).

[70] M. Aaboud et al. (ATLAS Collaboration), Search for dark matter and other new phenomena in events with an energetic jet and large missing transverse momentum using the ATLAS detector, J. High Energy Phys. 01 (2018) 126.

[71] M. Aaboud et al. (ATLAS Collaboration), Search for squarks and gluinos in final states with jets and missing transverse momentum using $36 \mathrm{fb}^{-1}$ of $\sqrt{s}=13 \mathrm{TeV}$ pp collision data with the ATLAS detector, Phys. Rev. D 97, 112001 (2018).

[72] A. M. Sirunyan et al. (CMS Collaboration), Search for Evidence of the Type-III Seesaw Mechanism in Multilepton Final States in Proton-Proton Collisions at $\sqrt{s}=13 \mathrm{TeV}$, Phys. Rev. Lett. 119, 221802 (2017).

[73] CMS Collaboration, Search for vector-like leptons in multilepton final states in pp collisions at $\sqrt{s}=13 \mathrm{TeV}$, Report No. CMS-PAS-EXO-18-005.

[74] M. Aaboud et al. (ATLAS Collaboration), Search for supersymmetry in events with four or more leptons in $\sqrt{s}=$ $13 \mathrm{TeV} p p$ collisions with ATLAS, Phys. Rev. D 98, 032009 (2018). 